\title{
INVARIANT TENSOR FIELDS AND ORBIT VARIETIES FOR FINITE ALGEBRAIC TRANSFORMATION GROUPS
}

\author{
Mark Losik, Peter W. Michor, Vladimir L. Popov \\ To C. S. Seshadri on the occasion of his 70th birthday
}

\begin{abstract}
Let $X$ be a smooth algebraic variety endowed with an action of a finite group $G$ such that there exists a geometric quotient $\pi_{X}: X \rightarrow X / G$. We characterize rational tensor fields $\tau$ on $X / G$ such that the pull back of $\tau$ is regular on $X$ : these are precisely all $\tau$ such that $\operatorname{div}_{R_{X / G}}(\tau) \geqslant 0$ where $R_{X / G}$ is the reflection divisor of $X / G$ and $\operatorname{div}_{R_{X / G}}(\tau)$ is the $R_{X / G}$-divisor of $\tau$. We give some applications, in particular to a generalization of Solomon's theorem. In the last section we show that if $V$ is a finite dimensional vector space and $G$ a finite subgroup of $\mathrm{GL}(V)$, then each automorphism $\psi$ of $V / G$ admits a biregular lift $\varphi: V \rightarrow V$ provided that $\psi$ maps the regular stratum to itself and $\psi_{*}\left(R_{X / G}\right)=R_{X / G}$.
\end{abstract}

\section{Introduction}

Let $X$ be a smooth algebraic variety endowed with an action of a finite group $G$. Assume that there exists a geometric quotient $\pi_{X}: X \rightarrow X / G$ (this is always the case if $X$ is quasi-projective, cf. Subsection 2.4).

In this paper we study the interrelations between rational tensor fields on $X$ and $X / G$. If $\tau$ is a rational tensor field on $X / G$, we define a $G$-invariant rational tensor field $\pi_{X}^{*}(\tau)$ on $X$ called the pull back of $\tau$. If $\theta$ is a $G$-invariant rational tensor field on $X$, we define a rational tensor field $\pi_{X *}(\theta)$ on $X / G$ called the push forward of $\theta$. We have $\pi_{X *}\left(\pi_{X}^{*}(\tau)\right)=\tau$ and $\pi_{X}^{*}\left(\pi_{X *}(\theta)\right)=\theta$.

Given this, we consider the following problem: Let $\tau$ be a rational tensor field on $X / G$. When is the pull back $\pi_{X}^{*}(\tau)$ regular on $X$ ?

To that end we consider the Luna stratification of $X / G$. Let $(X / G)_{1}$ be the union of all codimension 1 strata. We show that $(X / G)_{1}$ is contained in the smooth locus of $X / G$. Let $(X / G)_{1}=(X / G)_{1}^{1} \cup \ldots \cup(X / G)_{1}^{d}$ be the

1991 Mathematics Subject Classification. 14L24, 14L30.

Key words and phrases. Finite group, orbit, tensor field, orbit space, lifting.

M.L. and P.W.M. were supported by "Fonds zur Förderung der wissenschaftlichen Forschung, Projekt P 14195 MAT". 
decomposition into irreducible components. We show that for any $l=1, \ldots, d$ and $z \in(X / G)_{1}^{l}, x \in \pi_{X}^{-1}(z)$ the stabilizer of $x$ is a cyclic group whose image under the slice representation is generated by a pseudo-reflection of order $r_{l}$ depending only on $l$. We encode this information into the reflection divisor $R_{X / G}:=r_{1} R_{1}+\ldots+r_{d} R_{d} \in \operatorname{Div}(X / G)$ where $R_{l}$ is the prime divisor whose support is the closure of $(X / G)_{1}^{l}$. Further, for any nonzero rational tensor field $\tau$ on $X / G$ and positive divisor $B \in \operatorname{Div}(X / G)$ we $\operatorname{define} \operatorname{div}(\tau) \in \operatorname{Div}(X / G)$, the divisor of $\tau$, and $\operatorname{div}_{B}(\tau) \in \operatorname{Div}(X / G)$, the $B$-divisor of $\tau$. Locally $\operatorname{div}(\tau)$ is the minimum of divisors of the component functions of $\tau$ in the framings of tangent and cotangent bundles. The $\operatorname{divisor} \operatorname{div}_{B}(\tau)$ is obtained from $\operatorname{div}(\tau)$ by means of some "modification along" $B$ (see Subsection 3.6).

Our main result is that $\pi_{X}^{*}(\tau)$ is regular on $X$ if and only if $\operatorname{div}_{R_{X / G}}(\tau) \geqslant 0$.

As a corollary we obtain a generalization of Solomon's theorem [So], and prove that the push forward of a $G$-invariant regular tensor field on $X$ that is skew symmetric with respect to the covariant entries is regular on the smooth locus of $X / G$.

Another application pertains to the case where $X$ is a vector space $V$ with a linear action of $G$ : we obtain a characterization of $G$-invariant polynomials on $V^{\oplus s}$ in terms of rational multi-symmetric covariant tensor fields on $V / G$.

In the last section we prove that any automorphism $\psi$ of the algebraic variety $V / G$ such that $\psi\left((V / G)_{0}\right) \subseteq(V / G)_{0}$ and $\psi_{*}\left(R_{V / G}\right)=R_{V / G}$ can be lifted to an automorphism of the algebraic variety $V$. The proof is based on the relevant result in the analytic setting, $[\mathrm{KLM}]$, so what we really prove is that analytic lift is actually algebraic.

Throughout in this paper we assume that the base field $k$ is algebraically closed of characteristic 0 . In the last section we use the result from [KLM] that is proved for $k=\mathbb{C}$, so passing to the general case is carried out by Lefschetz's principle. However note that our proofs of the results from Sections 2-4 could be extended mutatis mutandis to the case when char $k$ is positive and subject to some non-divisibility and magnitude conditions; for this purpose one should use the relevant replacement of the slice theorem [Lu] proved in $[\mathrm{BR}]$.

This paper is the algebraic geometrical companion of paper [KLM] where similar results were obtained for analytic actions of finite groups.

We thank Yu. Neretin for helpful discussions. Thanks are also due to the referee for suggestions and comments which has led to eliminating some inaccuracies and improvement in the exposition.

Notation, terminology and conventions:

- $\mathcal{O}_{x, X}$ and $T_{x, X}$ are respectively the local ring and tangent space at a point $x$ of an algebraic variety $X$.

- $X_{\mathrm{sm}}$ is the smooth locus of $X$.

- $\operatorname{Div}(X)$ is the Weil divisor group of $X$.

- $\operatorname{div}(f)$ is the divisor of a rational function $f$.

- $\operatorname{supp}(D)$ is the support of $D \in \operatorname{Div}(X)$. 
- A positive divisor is called prime if it not sum of two positive divisors.

- $m_{C, D}$ is the multiplicity of a prime divisor $C$ in a divisor $D$.

- $d_{x} \varphi$ is the differential of a morphism $\varphi$ at a point $x$.

- $G \cdot z$ and $G_{z}$ are respectively the orbit and stabilizer of a point $z$ of a set $Z$ endowed with an action of a group $G$.

- $Z^{S}:=\{z \in Z \mid g \cdot z=z$ for all $g \in S\}$.

- $|S|$ is the number of elements of a finite set $S$.

All group actions considered in this paper are algebraic actions on algebraic varieties.

\section{Preliminaries}

2.1. Excellent morphisms. Recall that a morphism of algebraic varieties $\varphi: X \rightarrow Y$ is called étale at a point $x \in X$ if the homomorphism of local rings $\varphi^{*}: \mathcal{O}_{\varphi(x), Y} \rightarrow \mathcal{O}_{x, X}$ induces the isomorphism of their completions. If $x \in X_{\mathrm{sm}}$ and $\varphi(x) \in Y_{\mathrm{sm}}$, then $\varphi$ is étale at $x$ iff $d_{x} \varphi: T_{x, X} \rightarrow T_{\varphi(x), Y}$ is an isomorphism. If $\varphi$ is étale at each point of $X$, it is called étale.

Let $G$ be a reductive algebraic group. We refer to [PV] regarding the following basic facts.

Let $X$ be an affine algebraic $G$-variety. We denote by $X / / G$ the categorical quotient of $X$ by $G$, i.e., the affine algebraic variety whose algebra of regular functions is $k[X]^{G}$ (it is finitely generated by Hilbert's theorem). We denote by $\pi_{X}=\pi_{X, G}: X \rightarrow X / / G$ the canonical projection (induced by the inclusion $\left.k[X]^{G} \hookrightarrow k[X]\right)$.

Every fiber of $\pi_{X}$ contains a unique closed orbit. For a point $x \in X$, if the orbit $G \cdot x$ is closed in $X$, then the stabilizer $G_{x}$ is reductive. If $G$ is finite, then $\pi_{X}: X \rightarrow X / / G$ is a geometric quotient and we denote $X / / G$ by $X / G$.

Let $\varphi: X \rightarrow Y$ be a $G$-morphism of affine $G$-varieties. Then we have the following commutative diagram

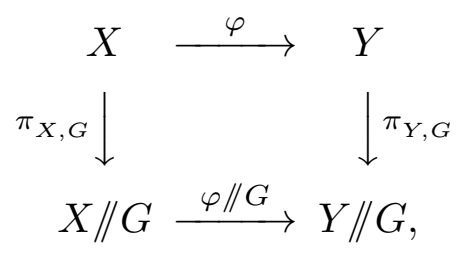

where $\varphi / / G$ is induced by the restriction of $\varphi^{*}: k[Y] \rightarrow k[X]$ to $k[Y]^{G}$ (if $G$ is finite, we denote $\varphi / / G$ by $\varphi / G)$. If $\varphi / / G$ is étale and (2.1.1) is the base change diagram (so that $X=X / / G \times_{Y / / G} Y$ ), then $\varphi$ is called excellent. In this case, $\varphi$ itself is étale. If $\varphi$ is excellent, then for any $b \in X / / G$ the restriction of $\varphi$ yields a $G$-isomorphism $\pi_{X}^{-1}(b) \rightarrow \pi_{Y}^{-1}((\varphi / / G)(b))$. Hence if $Y$ is irreducible and $\varphi$ is excellent, then $G$ acts faithfully on $X$ iff $G$ acts faithfully on $Y$.

2.2. Étale slices. The following theorem is proved in $[\mathrm{Lu}]$. 
Theorem 2.2.1. Let $X$ be an affine algebraic G-variety for a reductive algebraic group $G$, and let $x \in X$ be a point such that the orbit $G \cdot x$ is closed in $X$.

(i) There is a $G_{x^{-}}$stable locally closed affine subvariety $S \subseteq X$, called étale slice at $x$, such that $x \in S$ and the $G$-morphism

$$
\sigma_{S}: G *_{G_{x}} S \rightarrow X
$$

induced by $G \times S \rightarrow X,(g, s) \mapsto g \cdot s$, is excellent.

(ii) If $x \in X_{\mathrm{sm}}$, there exist a $\pi_{S, G_{x}}$-saturated affine open neighborhood $U$ of $x$ in $S$ and a $G_{x}$-equivariant excellent morphism

$$
\lambda_{x}: U \rightarrow T_{x, S}
$$

Here by $G *_{G_{x}} S$ we denote the homogeneous fiber space over $G / G_{x}$ with the fiber $S$, i.e., the categorical quotient of $G \times S$ by the $G_{x^{-}}$action $h \cdot(g, s)=$ $\left(g h^{-1}, h \cdot s\right)$ (actually in this case it is the geometrical quotient).

Theorem 2.2.1 is crucial for this paper. We will apply it only in the setting of finite group actions, in which case its proof is simpler than in general one. Therefore for making the paper more self-contained, in short Appendix at the end of the paper we give, following the referee's suggestion, some details of the proof of Theorem 2.2.1 for finite $G$.

2.3. The Luna stratification. Let $G$ be a reductive algebraic group. Denote by $\mathcal{V}(G)$ the set of isomorphism classes of all algebraic vector $G$-bundles over homogeneous spaces $G / H$ where $H$ ranges over all reductive subgroups of $G$.

Let $X$ be an affine algebraic $G$-variety. For a point $b \in X / / G$, let $G \cdot x$ be the unique closed orbit in $\pi_{X}^{-1}(b)$. Since $G_{x}$ is reductive, there is a $G_{x}$-stable direct linear complement $N_{x}$ to $T_{x, G \cdot x}$ in $T_{x, X}$. The $G$-bundle $G *_{G_{x}} N_{x} \rightarrow$ $G / G_{x}$, called the normal bundle of $G \cdot x$, is the representative of some element $\nu(b) \in \mathcal{V}(G)$. In this way we get a mapping $\nu: X / / G \rightarrow \mathcal{V}(G), b \mapsto \nu(b)$.

Let $s \in \mathcal{V}(G)$. Then the sets $(X / / G)_{s}:=\nu^{-1}(s)$ and $X_{s}:=\pi_{X}^{-1}\left((X / / G)_{s}\right)$ are called the strata of type $s$ of $X / / G$ and $X$ respectively.

The following theorem is proved in $[\mathrm{Lu}]$.

Theorem 2.3.1. Retain the above notation and assume that $X$ is smooth. Then

(i) The image of $\nu$ in $\mathcal{V}(G)$ is finite.

(ii) Each stratum $(X / / G)_{s}$ is a smooth locally closed subvariety of $X / / G$.

(iii) Each stratum $X_{s}$ is a locally closed smooth $\pi_{X}$-saturated subvariety of $X$. There is a G-variety $F_{s}$ such that each fiber $\pi_{X}^{-1}(b), b \in(X / / G)_{s}$, is $G$-isomorphic to $F_{s}$ and the restriction $\left.\pi_{X}\right|_{X_{s}}: X_{s} \rightarrow(X / / G)_{s}$ is a fiber bundle with the fiber $F_{s}$ locally trivial in étale topology (i.e., each point of $(X / / G)_{s}$ has an open neighborhood $U$ such that the pull back of this bundle over a suitable étale covering of $U$ becomes trivial). 
If $\varphi: X \rightarrow Y$ is an excellent morphism of smooth irreducible affine algebraic $G$-varieties, then $X_{s}=\varphi^{-1}\left(Y_{s}\right)$ and $(X / / G)_{s}=(\varphi / / G)^{-1}\left((Y / / G)_{s}\right)$ for every $s \in \mathcal{V}(G)$.

2.4. Quotients by finite group actions. Now assume that $G$ is a finite group and $X$ an algebraic $G$-variety (not necessarily affine). Consider the property

(Q) Every $G$-orbit is contained in an affine open subset of $X$.

For instance, $(Q)$ holds if $X$ is quasi-projective.

Then one has the following criterion.

Theorem 2.4.1. The following properties are equivalent:

(i) (Q) holds.

(ii) There exists a geometric quotient

$$
\pi_{X}=\pi_{X, G}: X \rightarrow X / G
$$

The morphism $\pi_{X}$ is automatically affine (i.e., for any open affine subset $U$ of $X / G$ the set $\pi_{X}^{-1}(U)$ is affine).

Proof. See [PV, Theorem 4.14].

Assume that there exists a geometric quotient (2.4.1). Take a point $x \in X$. As the group $G$ is finite, the orbit $G \cdot x$ is closed. As the morphism $\pi_{X}$ is affine, the orbit $G \cdot x$ is contained in a $G$-stable affine open subset of $X$. Hence Theorem 2.2.1 is applicable to the action of $G$ on this subset. This yields

Theorem 2.4.2. Assume that for an action of a finite group $G$ on an algebraic variety $X$ there exists a geometric quotient (2.4.1). Then statements (i) and (ii) of Theorem 2.2 hold.

We retain the notation of Subsection 2.3. For any point $b \in X / G$, the fiber $\pi_{X}^{-1}(b)$ is an orbit $G \cdot x$. As it is finite, $N_{x}=T_{x, X}$, so $\nu(b)$ is the class of $G *_{G_{x}} T_{x, X}$. Like in Subsection 2.3, we consider the mapping $\nu: X / G \rightarrow \mathcal{V}(G)$, $b \mapsto \nu(b)$, and for any $s \in \mathcal{V}(G)$ define the sets $(X / G)_{s}:=\nu^{-1}(s)$ and $X_{s}:=$ $\pi_{X}^{-1}\left((X / G)_{s}\right)$. We call them the strata of type $s$ of $X / G$ and $X$ respectively. As every orbit is contained in a $G$-stable affine open subset of $X$, applying Theorem 2.3.1 we obtain the following

Theorem 2.4.3. Assume that for an action of a finite group $G$ on a smooth algebraic variety $X$ there exists a geometric quotient (2.4.1). Then statements (i), (ii) and (iii) of Theorem 2.3 hold.

The definition of strata yields the following description. Let $H$ be the stabilizer of a point in $X$, and let $M$ be a finite dimensional algebraic $H$-module over $k$. Then

$$
G \cdot\left\{x \in X^{H} \mid G_{x}=H, \text { and the } H \text {-modules } T_{x, X} \text { and } M \text { are isomorphic }\right\}
$$


is a (possibly empty) stratum $X_{s}$, where $s$ is the class of $G{ }^{*}{ }_{H} M$, and each stratum of $X$ is obtained in this way. If $X_{s}$ is nonempty, $Z$ is an irreducible component of $X_{s}$, and $z$ is a point of $Z$, then existence of morphism (2.2.2) implies that $\operatorname{dim} Z=\operatorname{dim} M^{H}$ and $Z \subseteq X^{G_{z}}$.

We denote by $(X / G)_{i}$, resp. $X_{i}$, the union of all strata of $X / G$, resp. $X$, of codimension $i$. This is a locally closed smooth subvariety of $X / G$, resp. $X$. We have $X_{i}=\pi_{X}^{-1}\left((X / G)_{i}\right)$. The subvariety $(X / G)_{0}$, resp. $X_{0}$, is a single stratum of $X / G$, resp. $X$. It is called the principal stratum and is characterized among all $(X / G)_{i}$, resp. $X_{i}$, by the property that it is dense and open in $X / G$, resp. $X$. We have the inclusion $(X / G)_{0} \subseteq(X / G)_{\mathrm{sm}}$. If $X$ is irreducible, $X_{0}$ coincides with the open set of all points whose stabilizer is the kernel of action; in particular if the action is faithful, $X_{0}$ is the open set of all points with trivial stabilizers. By Theorem 2.4.2, the map $\left.\pi_{X}\right|_{X_{0}}: X_{0} \rightarrow(X / G)_{0}$ is étale.

2.5. Local description of the codimension 1 strata. The following lemma is a basic tool of our analysis of pull backs of tensor fields on quotient varieties. It shows that, in étale topology, a neighborhood of a point of a codimension 1 stratum of any quotient variety coincides with such a neighborhood for a linear action of a finite cyclic group generated by pseudo-reflection.

Lemma 2.5.1. Assume that for a faithful action of a finite group $G$ on an irreducible smooth algebraic variety $X$ there exists a geometric quotient (2.4.1). Let $x$ be a point of $X_{1}$. Then:

(i) $G_{x}$ is a finite cyclic group.

(ii) There are a character $\chi: G_{x} \rightarrow k^{\times}$of order $\left|G_{x}\right|$ and the local parameters $u_{1}, \ldots, u_{n}$ of $X$ at $x$ such that $u_{n}$ is a local equation of $X_{1}$ at $x$, each $u_{1}, \ldots, u_{n-1}$ is $G_{x}$-invariant, and $g \cdot u_{n}=\chi(g) u_{n}$ for all $g \in G_{x}$.

(iii) $\pi_{X, G}(x) \in(X / G)_{\mathrm{sm}}$.

(iv) $y:=\pi_{X, G_{x}}(x) \in\left(X / G_{x}\right)_{\mathrm{sm}}$.

(v) There are the local parameters $v_{1}, \ldots, v_{n}$ of $X / G_{x}$ at $y$ such that

$$
\pi_{X, G_{x}}^{*}\left(v_{1}\right)=u_{1}, \ldots, \pi_{X, G_{x}}^{*}\left(v_{n-1}\right)=u_{n-1}, \pi_{X, G_{x}}^{*}\left(v_{n}\right)=u_{n}^{\left|G_{x}\right|} .
$$

Proof. By Theorem 2.4.2, there is an étale slice $S$ of $X$ at $x$. As $G$ is finite, $S$ is a $G_{x}$-stable neighborhood of $x$. Hence the set $U$ in $(2.2 .2)$ is a $G_{x}$-stable neighborhood of $x$ as well. Since $X$ is irreducible, $U$ is dense in $X$. As the action of $G$ on $X$ is faithful, this implies that the action of $G_{x}$ on $U$ is faithful as well.

It follows from smoothness of $X_{1}$ and $X$ that $T_{x, X_{1}}$ is a hyperplane in $T_{x, X}$. Since the irreducible component of $X_{1}$ containing $x$ lays in $X^{G_{x}}$, we have $T_{x, X_{1}} \subseteq T_{x, X}^{G_{x}}$. As the action of $G_{x}$ on $U$ is faithful and morphism (2.2.2) is excellent, the action of $G_{x}$ on $T_{x, S}=T_{x, X}$ is faithful as well. As there is a one-dimensional $G_{x}$-stable direct complement to $T_{x, X_{1}}$ in $T_{x, X}$, this implies that $T_{x, X_{1}}=T_{x, X}^{G_{x}}$, and $G_{x}$ is a cyclic group of order $\left|G_{x}\right|$ whose generator acts on $T_{x, X}$ as a pseudo-reflection with mirror $T_{x, X_{1}}$. 
From this we deduce that there are a character $\chi: G_{x} \rightarrow k^{\times}$of order $\left|G_{x}\right|$, and a basis $t_{1}, \ldots, t_{n}$ of $T_{x, X}^{*}$ such that $t_{1}, \ldots, t_{n-1}$ are $G_{x}$-invariant and $t_{n}$ is a $G_{x}$-semi-invariant with weight $\chi$. The sequence $t_{1}, \ldots, t_{n}$ is a system of local parameters of $T_{x, X}$ at 0 , and $t_{n}$ is a local equation of $T_{x, X_{1}}$ at 0 . Since the morphism (2.2.2) is étale,

$$
u_{1}:=\lambda_{x}^{*}\left(t_{1}\right), \ldots, u_{n}:=\lambda_{x}^{*}\left(t_{n}\right) \in k[U]
$$

is a system of local parameters at $x$ that has the properties stated in (ii).

We have $k\left[T_{x, X}\right]=k\left[t_{1}, \ldots, t_{n}\right]$. Hence $k\left[T_{x, X}\right]^{G_{x}}$ is the polynomial algebra in $t_{1}, \ldots, t_{n-1}, t_{n}^{\left|G_{x}\right|}$, and $T_{x, X} / G_{x}$ is an affine space.

The set $\left(G *_{G_{x}} U\right) / G$ is open in $\left(G *_{G_{x}} S\right) / G$. By Theorem 2.4.2, we have the following étale morphisms

$$
X / G \stackrel{\sigma_{S} / G}{\longleftarrow}\left(G \times_{G_{x}} S\right) / G \hookleftarrow\left(G \times_{G_{x}} U\right) / G=U / G_{x} \stackrel{\lambda_{x} / G_{x}}{\longrightarrow} T_{x, X} / G_{x} .
$$

Thus the following commutative diagram arises:

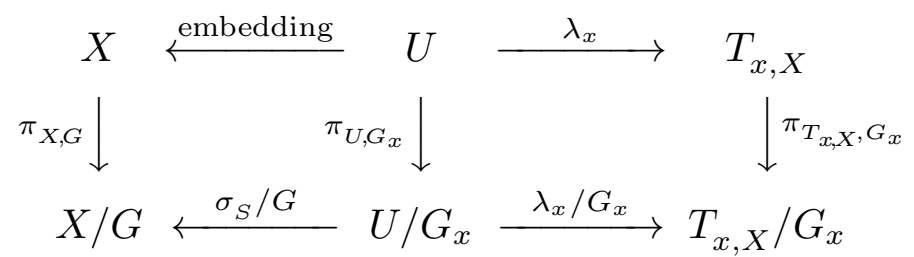

As $\pi_{T_{x, X}, G_{x}}(0) \in\left(T_{x, X} / G_{x}\right)_{\mathrm{sm}}$ and $U / G_{x}=\pi_{X, G_{x}}(U)$ is a neighborhood of $y$ in $X / G_{x}$, this yields $y \in\left(X / G_{x}\right)_{\mathrm{sm}}$ and $\pi_{X, G}(x)=\left(\sigma_{S} / G\right)(y) \in(X / G)_{\mathrm{sm}}$.

By our construction, $k\left[T_{x, X} / G_{x}\right]=k\left[w_{1}, \ldots, w_{n}\right]$ where $w_{1} \ldots, w_{n}$ are defined by the equalities

$$
\pi_{T_{x, X}}^{*}\left(w_{1}\right)=t_{1}, \ldots, \pi_{T_{x, X}}^{*}\left(w_{n-1}\right)=t_{n-1}, \pi_{T_{x, X}}^{*}\left(w_{n}\right)=t_{n}^{\left|G_{x}\right|}
$$

Since $\lambda_{x} / G_{x}$ is étale, $v_{1}:=\left(\lambda_{x} / G_{x}\right)^{*}\left(w_{1}\right), \ldots, v_{n}:=\left(\lambda_{x} / G_{x}\right)^{*}\left(w_{n}\right)$ is a system of parameters of $X / G_{x}$ at $y$. It follows from (2.5.2) that

$$
\pi_{U, G_{x}}^{*}\left(v_{1}\right)=u_{1}, \ldots, \pi_{U, G_{x}}^{*}\left(v_{n-1}\right)=u_{n-1}, \pi_{U, G_{x}}^{*}\left(v_{n}\right)=u_{n}^{\left|G_{x}\right|}
$$

2.6. Reflection divisor of quotient variety. Let $X$ be an irreducible smooth algebraic variety endowed with a faithful action of a finite group $G$ such that there exists a geometric quotient (2.4.1). As $X$ is normal, $X / G$ is normal as well, [PV, §4 and Theorem 3.16].

It follows from the discussion in Subsection 2.4 that if $(X / G)_{1} \neq \varnothing$ and

$$
(X / G)_{1}=(X / G)_{1}^{1} \cup \ldots \cup(X / G)_{1}^{d}
$$


is the decomposition into irreducible components, then for each $l=1, \ldots, d$ and $x \in \pi_{X}^{-1}(b), b \in(X / G)_{1}^{l}$ the integer $\left|G_{x}\right|$ does not depend on $b$ and $x$. Denote it by $r_{l}$.

We encode the information about $r_{1}, \ldots, r_{d}$ into the divisor

$$
R_{X / G}:=r_{1} R_{1}+\ldots+r_{d} R_{d} \in \operatorname{Div}(X / G),
$$

where $R_{l} \in \operatorname{Div}(X / G)$ is the prime divisor such that $\operatorname{supp}\left(R_{l}\right)$ is the closure of $(X / G)_{1}^{l}$.

If $(X / G)_{1}=\varnothing$, we put $R_{X / G}=0$. Thus

$$
R_{X / G}=0 \Longleftrightarrow(X / G)_{1}=\varnothing .
$$

We call $R_{X / G}$ the reflection divisor of $X / G$. We remark that

$$
(X / G)_{1}^{i} \cap(X / G)_{1}^{j}=\varnothing \text { if } i \neq j .
$$

because of smoothness of $(X / G)_{1}$.

\section{Lifting Tensor Fields}

3.1. Rational tensor fields on algebraic varieties. Let $Y$ be an irreducible algebraic variety. Denote by $T\left(Y_{\mathrm{sm}}\right)$, resp. $T^{*}\left(Y_{\mathrm{sm}}\right)$, the tangent, resp. cotangent, bundle of $Y_{\mathrm{sm}}$. We define a rational tensor field of type $\left(\begin{array}{l}p \\ q\end{array}\right)$ on $Y$ as a rational section of the tensor product of $p$ copies of $T\left(Y_{\mathrm{sm}}\right)$ and $q$ copies of $T^{*}\left(Y_{\mathrm{sm}}\right)$ whose factors are taken in a fixed order. We call $p+q$ the valency of such a section. As changing of the order yields an isomorphic vector bundle, we consider only the following representative of this class of vector bundles:

$$
T_{q}^{p}\left(Y_{\mathrm{sm}}\right):=T\left(Y_{\mathrm{sm}}\right)^{\otimes p} \otimes T^{*}\left(Y_{\mathrm{sm}}\right)^{\otimes q} .
$$

The set of points where a rational tensor field $\tau \in \Gamma_{\text {rat }}\left(T_{q}^{p}\left(Y_{\mathrm{sm}}\right)\right)$ is defined is an open subset of $Y_{\mathrm{sm}}$. We denote it by $\operatorname{dom}(\tau)$. We say that $\tau$ is regular on a subset $U$ of $X$ if $U \subseteq \operatorname{dom}(\tau)$. For a point $y \in \operatorname{dom}(\tau)$, we denote by $\tau(y)$ the value of $\tau$ at $y$.

The set $\Gamma_{\mathrm{rat}}\left(T_{q}^{p}\left(Y_{\mathrm{sm}}\right)\right)$ of rational tensor fields of type $\left(\begin{array}{l}p \\ q\end{array}\right)$ on $Y$ has a natural structure of $k(Y)$-module. A rational tensor field $\nu$ of type $\left(\begin{array}{l}1 \\ 0\end{array}\right)$ on $Y$ is a rational vector field on $Y_{\mathrm{sm}}$ or, equivalently, a derivation of the $k$-algebra $k(Y)$. A rational tensor field $\omega$ on $Y$ of type $\left(\begin{array}{l}0 \\ 1\end{array}\right)$ is a rational differential 1-form on $Y_{\mathrm{sm}}$. We denote by $\omega(\nu)$ the rational function on $Y$ whose value at a point $y \in \operatorname{dom}(\omega) \cap \operatorname{dom}(\nu)$ is equal to the value of $\omega(y)$ at $\nu(y)$. Rational sections of $\wedge^{q}\left(Y_{\mathrm{sm}}\right)$ are rational differential $q$-forms on $Y_{\mathrm{sm}}$. Rational differential $q$-forms on $Y$ defined in [Sh] are naturally identified with them by means of restriction to $Y_{\mathrm{sm}}$.

Let $v_{1}, \ldots, v_{n}$ be a system of local parameters of $Y_{\mathrm{sm}}$ at a point $y \in Y_{\mathrm{sm}}$. Then there is a neighborhood $V$ of $y$ in $Y_{\mathrm{sm}}$ such that $v_{1}, \ldots, v_{n} \in k[V]$ and 
$d v_{1}(a), \ldots, d v_{n}(a)$ is a basis of $T_{a, V}^{*}$ for each point $a \in V$, [Sh, Ch. III, §5]. Let $\frac{\partial}{\partial v_{1}}, \ldots, \frac{\partial}{\partial v_{n}}$ be the regular vector fields on $V$ defined by conditions

$$
d v_{i}\left(\frac{\partial}{\partial v_{j}}\right)=\delta_{i j}
$$

Then the (numbered) set of tensors $\left(\frac{\partial}{\partial v_{i_{1}}} \otimes \cdots \otimes \frac{\partial}{\partial v_{i_{p}}} \otimes d v_{j_{1}} \otimes \cdots \otimes d v_{j_{q}}\right)(a)$ for each point $a \in V$ is a basis of $T_{a, V}^{\otimes p} \otimes T_{a, V}^{* \otimes q}$.

This implies that for each rational tensor field $\tau \in \Gamma_{\text {rat }}\left(T_{q}^{p}\left(Y_{\mathrm{sm}}\right)\right)$ there is a unique decomposition

$$
\tau=\sum_{i_{1}, \ldots, i_{p}, j_{1}, \ldots, j_{q}} \tau_{j_{1} \ldots j_{q}}^{i_{1} \ldots i_{p}} \frac{\partial}{\partial v_{i_{1}}} \otimes \cdots \otimes \frac{\partial}{\partial v_{i_{p}}} \otimes d v_{j_{1}} \otimes \cdots \otimes d v_{j_{q}}
$$

where $\tau_{j_{1} \ldots j_{q}}^{i_{1} \ldots i_{p}} \in k(Y)$, and we have

$$
V \cap \operatorname{dom}(\tau):=\left\{a \in V \mid \text { each } \tau_{j_{1} \ldots j_{q}}^{i_{1} \ldots i_{p}} \text { is regular at } a\right\}
$$

¿From (3.1.2) we also obtain that for any point $a \in V$,

$$
\tau(a)=0 \Longleftrightarrow \tau_{j_{1} \ldots j_{q}}^{i_{1} \ldots i_{p}}(a)=0 \text { for all } i_{1}, \ldots, i_{p}, j_{1}, \ldots, j_{q}
$$

By (3.1.3), (3.1.4), the set of all zeros of $\tau$ is a closed subset of $\operatorname{dom}(\tau)$.

Now assume that the variety $Y$ is normal. If $\operatorname{dom}(\tau) \neq Y_{\text {sm }}$ and

$$
Y_{\mathrm{sm}} \backslash \operatorname{dom}(\tau)=\bigcup_{i=1}^{m}(\tau)_{\infty}^{i}
$$

is the decomposition of $Y_{\mathrm{sm}} \backslash \operatorname{dom}(\tau)$ into irreducible components, then by $(3.1 .3)$,

$$
\operatorname{codim}_{Y}(\tau)_{\infty}^{i}=1 \text { for all } i
$$

Thus if the "variety of poles" of a tensor field (i.e., the subvariety of $Y_{\mathrm{sm}}$ where this field is not defined) is nonempty, it is unmixed and of codimension 1. On the contrary, if $p$ or $q \neq 0$, then by (3.1.4), the variety of zeros of a tensor field of type $\left(\begin{array}{l}p \\ q\end{array}\right)$ can be mixed and/or of codimension greater than 1 .

3.2. Divisor of a rational tensor field. Let $\tau \in \Gamma_{\text {rat }}\left(T_{q}^{p}\left(Y_{\mathrm{sm}}\right)\right)$ be a nonzero rational tensor field. We $\operatorname{define} \operatorname{div}(\tau) \in \operatorname{Div}(Y)$, the divisor of $\tau$, as follows.

Let $C \in \operatorname{Div}(Y)$ be a prime divisor. Since $Y$ is normal, $\operatorname{supp}(C) \cap Y_{\text {sm }} \neq \varnothing$. Consider in $Y$ an open neighborhood $U$ of a point $y \in \operatorname{supp}(C) \cap Y_{\text {sm }}$ where $C$ is given by a local equation $t$. Then there is a unique integer $\nu(C, \tau)$ such that

(i) $\operatorname{supp}(C) \cap \operatorname{dom}\left(t^{-\nu(C, \tau)} \tau\right) \neq \varnothing$;

(ii) $t^{-\nu(C, \tau)} \tau$ does not identically vanish on $\operatorname{supp}(C) \cap \operatorname{dom}\left(t^{-\nu(C, \tau)} \tau\right)$. 
To see this, take $V$ and $v_{1}, \ldots, v_{n}$ as above assuming moreover that $V \subseteq U$ (this can be attained by replacing $V$ by $V \cap U)$. Thus (3.1.2) holds. Then (i) and (ii) hold iff there is a point $z \in \operatorname{supp}(C)$ such that all functions $t^{-\nu(C, \tau)} \tau_{j_{1} \ldots j_{q}}^{i_{1} \ldots i_{p}}$ are defined at $z$ but not all of them vanish at $z$. This unequivocally identifies $\nu(C, \tau)$ as

$$
\nu(C, \tau)=\min _{i_{1}, \ldots i_{p}, j_{1}, \ldots, j_{q}} m_{C, \operatorname{div}\left(\tau_{j_{1} \ldots j_{q}}^{i_{1} \ldots i_{p}}\right)} \cdot
$$

It is immediately seen that $\nu(C, \tau)$ is well defined (i.e., depends only on $C$ and $\tau$ but not on the choice of $V$ and $t)$, and that the set $\{C \mid \nu(C, \tau) \neq 0\}$ is finite (maybe empty). Now the divisor $\operatorname{div}(\tau)$ is defined by

$$
m_{C, \operatorname{div}(\tau)}:=\nu(C, \tau) \text { for all } C
$$

For $p=q=0$ this gives the usual definition of divisor of a rational function.

It follows from the definition and (3.1.5), (3.1.6) that if $m_{C, \operatorname{div}(\tau)}<0$, then $\operatorname{supp}(C)$ is the closure in $Y$ of one of the $(\tau)_{\infty}^{i}$ 's from (3.1.5). Whence if $\tau$ is a rational tensor field on $Y$, then

$$
\operatorname{div}(\tau) \geqslant 0 \Longleftrightarrow \tau \text { is regular on } Y_{\mathrm{sm}} \text {. }
$$

On the other hand, we remark that, unlike in the case of rational functions, in general $\operatorname{div}(\tau) \leqslant 0$ does not imply that rational tensor field $\tau$ vanishes nowhere.

3.3. Pull backs and push forwards of rational tensor fields. Consider a dominant rational map $\alpha: Z \rightarrow Y$ of irreducible algebraic varieties of the same dimension. As char $k=0$, the set $Z_{\alpha}$ of points of $Z_{\mathrm{sm}}$ where $\alpha$ is defined and étale is dense and open in $Z$, cf. [Sh, Ch. II, §6, no. 2, Lemma 2].

Consider a rational tensor field $\tau \in \Gamma_{\text {rat }}\left(T_{q}^{p}\left(Y_{\mathrm{sm}}\right)\right)$. As $d_{z} \alpha: T_{z, Z} \rightarrow T_{\alpha(z), Y}$ for $z \in Z_{\alpha}$ is an isomorphism, it yields an isomorphism

$$
\alpha_{z}^{*}:=\left(\left(d_{z} \alpha\right)^{-1}\right)^{\otimes p} \otimes\left(\left(d_{z} \alpha\right)^{*}\right)^{\otimes q}: T_{\alpha(z), Y}^{\otimes p} \otimes T_{\alpha(z), Y}^{* \otimes q} \rightarrow T_{z, Z}^{\otimes p} \otimes T_{z, Z}^{* \otimes q} .
$$

Hence for $W:=\alpha^{-1}(\operatorname{dom}(\tau)) \cap Z_{\alpha}$ we obtain the following section of $T_{q}^{p}(W)$ :

$$
\alpha^{*}(\tau): W \rightarrow T_{q}^{p}(W), \quad \alpha^{*}(\tau)(z):=\alpha_{z}^{*}(\tau(\alpha(z)))
$$

We claim that (3.3.2) is a rational tensor field on $Z$, regular on $W$. To show this, take a point $z \in W$, a system of local parameters $v_{1}, \ldots, v_{n}$ of $Y_{\mathrm{sm}}$ at $y=\alpha(z)$, and a neighborhood $V$ of $y$ in $\alpha(W)$ as in Subsection 3.1. Then conditions (3.1.1) define the rational vector fields $\frac{\partial}{\partial v_{j}}$ such that decomposition (3.1.2) holds. Put

$$
u_{i}:=\alpha^{*}\left(v_{i}\right)
$$


As $\alpha$ is étale et $z$, the sequence $u_{1}, \ldots, u_{n}$ is a system of parameters of $Z_{\mathrm{sm}}$ at $z$. Let $U$ be a neighborhood of $z$ in $Z_{\alpha}$ such that $\alpha(U) \subseteq V$. Then $u_{1}, \ldots, u_{n} \in k[U]$ and $d u_{1}(a), \ldots, d u_{n}(a)$ is a basis of $T_{z, U}^{*}$ for each point $a \in U$. Let $\frac{\partial}{\partial u_{1}}, \ldots, \frac{\partial}{\partial u_{n}}$ be the regular vector fields on $U$ defined by the conditions $d u_{i}\left(\frac{\partial}{\partial u_{j}}\right)=\delta_{i j}$. Then from (3.3.1), (3.3.2) and (3.1.1) we deduce that

$$
\alpha^{*}\left(d v_{i}\right)=d u_{i} \text { and } \alpha^{*}\left(\frac{\partial}{\partial v_{i}}\right)=\frac{\partial}{\partial u_{i}} \text { for all } i
$$

Hence by (3.1.2), there is a unique decomposition

$$
\alpha^{*}(\tau)=\sum_{i_{1}, \ldots, i_{p}, j_{1}, \ldots, j_{q}} \alpha^{*}\left(\tau_{j_{1} \ldots j_{q}}^{i_{1} \ldots i_{p}}\right) \frac{\partial}{\partial u_{i_{1}}} \otimes \cdots \otimes \frac{\partial}{\partial u_{i_{p}}} \otimes d u_{j_{1}} \otimes \cdots \otimes d u_{j_{q}}
$$

As $\tau_{j_{1} \ldots j_{q}}^{i_{1} \ldots i_{p}} \in k(Y)$, this shows that $\alpha^{*}(\tau)$ is a rational tensor field on $Z$, regular on $W$. We call $\alpha^{*}(\tau)$ the pull back of $\tau$. Thus we have a map

$$
\alpha^{*}: \Gamma_{\mathrm{rat}}\left(T_{q}^{p}\left(Y_{\mathrm{sm}}\right)\right) \rightarrow \Gamma_{\mathrm{rat}}\left(T_{q}^{p}\left(Z_{\mathrm{sm}}\right)\right), \quad \tau \mapsto \alpha^{*}(\tau)
$$

Now we consider the case where $Z$ is an irreducible smooth algebraic $G$-variety $X$ for a finite group $G$ such that there exists a geometric quotient (2.4.1), and $Y=X / G, \alpha=\pi_{X}$. The fixed point set of the natural action of $G$ on $\Gamma_{\text {rat }}\left(T_{q}^{p}\left(X_{\mathrm{sm}}\right)\right)$ is precisely the set of all $G$-invariant rational tensor fields of type $\left(\begin{array}{l}p \\ q\end{array}\right)$ on $X$.

If $\tau \in \Gamma_{\text {rat }}\left(T_{q}^{p}\left((X / G)_{\mathrm{sm}}\right)\right)$, then the definition implies that the field $\pi_{X}^{*}(\tau)$ is $G$-invariant. Thus we obtain a map

$$
\pi_{X}^{*}: \Gamma_{\text {rat }}\left(T_{q}^{p}\left((X / G)_{\mathrm{sm}}\right)\right) \rightarrow \Gamma_{\text {rat }}\left(T_{q}^{p}\left(X_{\mathrm{sm}}\right)\right)^{G}, \tau \mapsto \pi_{X}^{*}(\tau)
$$

We claim that $\pi_{X}^{*}$ is bijective. To prove this, take a field $\varphi \in \Gamma_{\text {rat }}\left(T_{q}^{p}\left(X_{\mathrm{sm}}\right)\right)^{G}$. As $\operatorname{dom}(\varphi)$ is a $G$-stable and open subset of $X$, and $X$ is an irreducible variety, $W:=\pi_{X}(\operatorname{dom}(\varphi)) \cap(X / G)_{0}$ is a dense open subset of $X / G$. Take points $b \in W$ and $x \in \pi_{X}^{-1}(b)$. As $\pi_{X}$ is étale at each point of $X_{0}$, we have an isomorphism $\left(\pi_{X}\right)_{x}^{*}$ (see $\left.(3.3 .1)\right)$. Since $\varphi$ is $G$-invariant, $\left(\left(\pi_{X}\right)_{x}^{*}\right)^{-1}(\varphi(x))$ depends only on $b$ but not on the choice of $x$ in $\pi_{X}^{-1}(b)$. Hence the following formula defines a section of $T_{q}^{p}(W)$ :

$$
\pi_{X *}(\varphi): W \rightarrow T_{q}^{p}(W), \quad \pi_{X *}(\varphi)(b)=\left(\left(\pi_{X}\right)_{x}^{*}\right)^{-1}(\varphi(x)) .
$$

We claim that $\pi_{X *}(\varphi)$ is a rational tensor field on $X / G$, regular on $W$. We call it the push forward of $\varphi$. The definitions imply that the maps $\pi_{X}^{*}$ and

$$
\pi_{X *}: \Gamma_{\mathrm{rat}}\left(T_{q}^{p}\left(X_{\mathrm{sm}}\right)\right)^{G} \rightarrow \Gamma_{\mathrm{rat}}\left(T_{q}^{p}\left((X / G)_{\mathrm{sm}}\right)\right), \varphi \mapsto \pi_{X *}(\varphi)
$$

are inverses of one another. 
In order to prove the latter claim, fix a system $v_{1}, \ldots, v_{n}$ of local parameters of $X / G$ at $b$, and let $V$ be a neighborhood of $b$ in $W$ such that $v_{1}, \ldots, v_{n} \in k[V]$ and $d v_{1}(b), \ldots, d v_{n}(b)$ is a basis of $T_{b, V}^{*}$ for each point $b \in V$. The set $U:=$ $\pi_{X}^{-1}(V)$ is an open $G$-stable neighborhood of $x$ in $\operatorname{dom}(\varphi) \cap X_{0}$. As $\left.\pi_{X}\right|_{X_{0}}$ is étale, $u_{1}=\pi_{X}^{*}\left(v_{1}\right), \ldots, u_{n}=\pi_{X}^{*}\left(v_{n}\right) \in k[U]^{G}$ is a system of local parameters of $X$ at $x$ such that $d u_{1}(a), \ldots, d u_{n}(a)$ is a basis of $T_{a, U}^{*}$ for each point $a \in U$. Hence there is a unique decomposition

$$
\varphi=\sum_{i_{1}, \ldots, i_{p}, j_{1}, \ldots, j_{q}} \varphi_{j_{1} \ldots j_{q}}^{i_{1} \ldots i_{p}} \frac{\partial}{\partial u_{i_{1}}} \otimes \cdots \otimes \frac{\partial}{\partial u_{i_{p}}} \otimes d u_{j_{1}} \otimes \cdots \otimes d u_{j_{q}}
$$

where $\varphi_{j_{1} \ldots j_{q}}^{i_{1} \ldots i_{p}} \in k(X)$ and $d u_{i}\left(\frac{\partial}{\partial u_{j}}\right)=\delta_{i j}$.

As the fields $\varphi, d u_{i}$ and $\frac{\partial}{\partial u_{i}}$ are $G$-invariant, uniqueness of (3.3.7) and the inclusion $\operatorname{dom}(\varphi) \supseteq U$ imply that all coefficients $\varphi_{j_{1} \ldots j_{q}}^{i_{1} \ldots i_{p}}$ in (3.3.7) are contained in $k[U]^{G}$. Hence, as $\pi_{X}: X \rightarrow X / G$ is a geometric quotient, there are functions $\psi_{j_{1} \ldots j_{q}}^{i_{1} \ldots i_{p}} \in k[V]$ such that

$$
\varphi_{j_{1} \ldots j_{q}}^{i_{1} \ldots i_{p}}=\pi_{X}^{*}\left(\psi_{j_{1} \ldots j_{q}}^{i_{1} \ldots i_{p}}\right)
$$

cf. $[\mathrm{PV}, \S 4]$. On the other hand, the definitions imply that for any point $a \in U$,

$$
\begin{aligned}
\left(\left(\pi_{X}^{*}\right)_{a}\right)^{-1}\left(\left(\frac{\partial}{\partial u_{i_{1}}} \otimes\right.\right. & \left.\left.\cdots \otimes \frac{\partial}{\partial u_{i_{p}}} \otimes d u_{j_{1}} \otimes \cdots \otimes d u_{j_{q}}\right)(a)\right) \\
& =\left(\frac{\partial}{\partial v_{i_{1}}} \otimes \cdots \otimes \frac{\partial}{\partial v_{i_{p}}} \otimes d v_{j_{1}} \otimes \cdots \otimes d v_{j_{q}}\right)\left(\pi_{X}(a)\right) .
\end{aligned}
$$

¿From (3.3.6)-(3.3.9), we deduce that the following decomposition holds

$$
\pi_{X *}(\varphi)=\sum_{i_{1}, \ldots, i_{p}, j_{1}, \ldots, j_{q}} \psi_{j_{1} \ldots j_{q}}^{i_{1} \ldots i_{p}} \frac{\partial}{\partial v_{i_{1}}} \otimes \cdots \otimes \frac{\partial}{\partial v_{i_{p}}} \otimes d v_{j_{1}} \otimes \cdots \otimes d v_{j_{q}}
$$

In turn, (3.3.10) yields that $\pi_{X *}(\varphi)$ is a rational tensor field of type $\left(\begin{array}{l}p \\ q\end{array}\right)$ on $X / G$, regular on $W$.

3.4. Regularity of the pull back of a tensor field on the quotient variety: Example. We retain the notation and conventions of the previous subsection. Our aim in Section 3 is to give a necessary and sufficient condition of regularity of the pull back of a rational tensor field on $X / G$. According to the discussion in Subsection 3.3, if $\pi_{X}^{*}(\tau)$ is regular on $X$, then $\tau$ is automatically regular on $(X / G)_{0}$. However in general this condition is not sufficient: tensor fields on $X / G$ with "bad singularities" along the irreducible components of $(X / G)_{1}$ may have pull backs that are not regular on the whole $X$. The following simplest example illustrates this phenomenon.

Example. Let $X$ be a one dimensional linear space over $k$. Let $G$ be a finite group acting on $X$ by $g \cdot a:=\chi(g) a$ where $\chi: G \rightarrow k^{\times}$is a character of order $r$. If $u$ is a nonzero linear function on $X$, then $k[X]=k[u]$ and 
$k[X]^{G}=k\left[u^{r}\right]$. Hence $X / G$ is an affine line with the coordinate function $v$, and $\pi_{X}:=\pi_{X, G}: X \rightarrow X / G$ is given by $\pi_{X}^{*}(v)=u^{r}$.

Each nonzero rational tensor field $\tau$ of type $\left(\begin{array}{l}p \\ q\end{array}\right)$ on $X / G$ has the form

$$
\tau=\alpha v^{m} \prod_{i=1}^{l}\left(v-\gamma_{i}\right)^{d_{i}}\left(\frac{\partial}{\partial v}\right)^{\otimes p} \otimes(d v)^{\otimes q},
$$

where $m, d_{i} \in \mathbb{Z}, \alpha, \gamma_{i} \in k \backslash\{0\}$ for all $i$, and $\gamma_{i} \neq \gamma_{j}$ for all $i \neq j$. Since $\pi_{X}^{*}(d v)=r u^{r-1} d u$ and $\pi_{X}^{*}\left(\frac{\partial}{\partial v}\right)=\frac{1}{r u^{r-1}} \frac{\partial}{\partial u}$, we have

$$
\begin{gathered}
\pi_{X}^{*}(\tau)=\pi_{X}^{*}(v)^{m} \prod_{i=1}^{l}\left(\pi_{X}^{*}(v)-\gamma_{i}\right)^{d_{i}}\left(\pi_{X}^{*}\left(\frac{\partial}{\partial v}\right)\right)^{\otimes p} \otimes\left(\pi_{X}^{*}(d v)\right)^{\otimes q} \\
=r^{q-p} u^{(r-1)(q-p)+r m} \prod_{i=1}^{l}\left(u^{r}-\gamma_{i}\right)^{d_{i}}\left(\frac{\partial}{\partial u}\right)^{\otimes p} \otimes(d u)^{\otimes q}
\end{gathered}
$$

Therefore the rational tensor field $\pi_{X}^{*}(\tau)$ is regular on $X$ if and only if

$$
\begin{aligned}
& \text { (i) } d_{i} \geqslant 0 \text { for all } i, \\
& \text { (ii) }(r-1)(q-p)+r m \geqslant 0 .
\end{aligned}
$$

For instance, if $r=3, m=-3, q-p=7$, and $d_{i}=0$ for all $i$, then $\tau$ is regular on $(X / G)_{0}$ but not regular on $X / G$, and $\pi_{X}^{*}(\tau)$ is regular on $X$. If $r=3, m=-5, q-p=7$ and $d_{i}=0$ for all $i$, then $\tau$ is regular on $(X / G)_{0}$, not regular on $X / G$, and $\pi_{X}^{*}(\tau)$ is not regular on $X$.

From the general criterion of regularity of the pull back that will be obtained below we will see that this example essentially elucidates the general mechanism governing the phenomenon under investigation.

3.5. Regularity of the pull back of a tensor field on a quotient variety: Case with no strata of codimension 1. The corollary of the following proposition shows that if there are no strata of codimension 1 , then the situation is simple and regularity of $\tau$ on $(X / G)_{0}$ is not only necessary but also sufficient for regularity of $\pi_{X}^{*}(\tau)$ on $X$.

Proposition 3.5.1. Let $X$ be an irreducible smooth algebraic variety endowed with an action of a finite group $G$. Assume that there exists a geometric quotient (2.4.1). Let $U$ be an open subset of $X / G$ such that $U \cap(X / G)_{1}=\varnothing$. Consider a tensor field $\tau \in \Gamma_{\text {rat }}\left(T_{q}^{p}\left((X / G)_{\mathrm{sm}}\right)\right)$ such that $U \cap(X / G)_{0} \subseteq \operatorname{dom}(\tau)$. Then $\pi_{X}^{-1}(U) \subseteq \operatorname{dom}\left(\pi_{X}^{*}(\tau)\right)$.

Proof. As $\operatorname{dom}(\tau) \supseteq U \cap(X / G)_{0}$, we have $\operatorname{dom}\left(\pi_{X}^{*}(\tau)\right) \supseteq \pi_{X}^{-1}(U) \cap X_{0}$. Since $U \cap(X / G)_{1}=\varnothing$, the complement of $\pi_{X}^{-1}(U) \cap X_{0}$ in $\pi_{X}^{-1}(U)$ has codimension $\geqslant 2$. Now the claim follows from (3.1.5) and (3.1.6).

Corollary 3.5.1. Assume moreover that $X$ contains no strata of codimension 1 . Then the pull back $\pi_{X}^{*}(\tau)$ of a rational tensor field $\tau$ on $X / G$ is regular on $X$ if and only if $\tau$ is regular on the principal stratum $(X / G)_{0}$.

Proof. As $(X / G)_{1}=\varnothing$, one can take $U=X / G$. 
3.6. Modifying the divisor of a tensor field by means of a nonnegative divisor. Let $Y$ be an irreducible normal algebraic variety and $B \in$ $\operatorname{Div}(Y)$ a nonnegative divisor. Consider a nonzero rational tensor field $\tau \in$ $\Gamma_{\text {rat }}\left(T_{q}^{p}\left(Y_{\mathrm{sm}}\right)\right)$. In this subsection we define a $\operatorname{divisor} \operatorname{div}_{B}(\tau) \in \operatorname{Div}(Y)$ that we call the $B$-divisor of $\tau$. It is obtained from $\operatorname{div}(\tau)$ by means of some "modification along" $B$. In the next subsection we will use this notion in our criterion of regularity of the pull back of a tensor field $\tau$ on the quotient variety $X / G$ : it is formulated in terms of $\operatorname{div}_{R_{X / G}}(\tau)$, where $R_{X / G}$ is the reflection divisor of $X / G$, see (2.6.2).

If $B=0$, we define

$$
\operatorname{div}_{0}(\tau):=\operatorname{div}(\tau)
$$

Now assume that $B>0$,

$$
B=b_{1} B_{1}+\ldots+b_{d} B_{d}
$$

where $B_{i}$ is a prime divisor and $b_{i}$ a positive integer for all $i$. Then $\operatorname{div}_{B}(\tau)$ is obtained from $\operatorname{div}(\tau)$ by modifying the multiplicities $m_{B_{1}, \operatorname{div}(\tau)}, \ldots, m_{B_{d}, \operatorname{div}(\tau)}$ as defined below.

To describe this modification, fix some $B_{l}$. As $\operatorname{codim}\left(\operatorname{supp}\left(B_{l}\right)\right)=1$ and $Y$ is normal, $\operatorname{supp}\left(B_{l}\right) \cap Y_{\text {sm }} \neq \varnothing$. Hence taking local parameters at a point $y \in \operatorname{supp}\left(B_{l}\right) \cap Y_{\mathrm{sm}}$, we can find an open neighborhood $V$ of $y$ in $Y_{\mathrm{sm}}$ and a system $v$ of functions $v_{1}, \ldots, v_{n} \in k[V] \operatorname{such}$ that $\operatorname{supp}\left(B_{l}\right) \cap V \neq \varnothing$, the ideal of $\operatorname{supp}\left(B_{l}\right) \cap V$ in $k[V]$ is generated by $v_{n}$, and $d v_{1}(a), \ldots, d v_{n}(a)$ is a basis of $T_{a, V}^{*}$ for each $a \in V$. Hence there is a unique decomposition

$$
\tau=\sum_{i_{1}, \ldots, i_{p}, j_{1}, \ldots, j_{q}} \delta_{j_{1} \ldots j_{q}}^{i_{1} \ldots i_{p}}
$$

where (see (3.1.2))

$$
\delta_{j_{1} \ldots j_{q}}^{i_{1} \ldots i_{p}}=\tau_{j_{1} \ldots j_{q}}^{i_{1} \ldots i_{p}} \frac{\partial}{\partial v_{i_{1}}} \otimes \cdots \otimes \frac{\partial}{\partial v_{i_{p}}} \otimes d v_{j_{1}} \otimes \cdots \otimes d v_{j_{q}}, \quad \tau_{j_{1} \ldots j_{q}}^{i_{1} \ldots i_{p}} \in k(Y) .
$$

With each nonzero tensor field $\delta_{j_{1} \ldots j_{q}}^{i_{1} \ldots i_{p}}$ we associate the following three integers $m, p^{\prime}$ and $q^{\prime}$ (depending on this field):

- $m:=m_{B_{l}, \operatorname{div}\left(\tau_{j_{1} \ldots j_{q}}^{i_{1} \ldots i_{p}}\right)}$;

- $p^{\prime}$ (resp. $\left.q^{\prime}\right)$ is the number of factors $\frac{\partial}{\partial v_{n}}$ (resp. $\left.d v_{n}\right)$ in the right-hand side of equality (3.6.4).

Now define

$$
\begin{gathered}
\mu\left(b_{l} B_{l}, \delta_{j_{1} \ldots j_{q}}^{i_{1} \ldots i_{p}}\right)_{V, v}:=\left(b_{l}-1\right)\left(q^{\prime}-p^{\prime}\right)+b_{l} m, \\
\varrho\left(b_{l} B_{l}, \tau\right)_{V, v}:=\min _{i_{1}, \ldots, i_{p}, j_{1} \ldots j_{q}} \mu\left(b_{l} B_{l}, \delta_{j_{1} \ldots j_{q}}^{i_{1} \ldots i_{p}}\right)_{V, v} .
\end{gathered}
$$


It easily follows from (3.6.3)-(3.6.6) that for any nonzero $f \in k(Y)$ and $\tau, \varsigma \in \Gamma_{\text {rat }}\left(T_{q}^{p}(Y)_{\mathrm{sm}}\right)$ such that $\tau+\varsigma \neq 0$ we have

$$
\begin{gathered}
\varrho\left(b_{l} B_{l}, f \tau\right)_{V, v}=b_{l} m_{B_{l}, \operatorname{div}(f)}+\varrho\left(b_{l} B_{l}, \tau\right)_{V, v}, \\
\varrho\left(b_{l} B_{l}, \tau+\varsigma\right)_{V, v} \geqslant \min \left\{\varrho\left(b_{l} B_{l}, \tau\right)_{V, v}, \varrho\left(b_{l} B_{l}, \varsigma\right)_{V, v}\right\} .
\end{gathered}
$$

Lemma 3.6.1. For any nonzero $\tau \in \Gamma_{\mathrm{rat}}\left(T_{q}^{p}(Y)_{\mathrm{sm}}\right), \varsigma \in \Gamma_{\mathrm{rat}}\left(T_{t}^{s}(Y)_{\mathrm{sm}}\right)$ we have

$$
\varrho\left(b_{l} B_{l}, \tau \otimes \varsigma\right)_{V, v}=\varrho\left(b_{l} B_{l}, \tau\right)_{V, v}+\varrho\left(b_{l} B_{l}, \varsigma\right)_{V, v} .
$$

Proof. If $\tau$ and $\varsigma$ are " monomials in $v$ ", i.e., $\tau=f \frac{\partial}{\partial v_{i_{1}}} \otimes \cdots \otimes \frac{\partial}{\partial v_{i_{p}}} \otimes d v_{j_{1}} \otimes \cdots \otimes d v_{j_{q}}$, $\varsigma=g \frac{\partial}{\partial v_{a_{1}}} \otimes \cdots \otimes \frac{\partial}{\partial v_{a_{s}}} \otimes d v_{b_{1}} \otimes \cdots \otimes d v_{b_{t}}$ where $f, g \in k(Y)$, then $\tau \otimes \varsigma$ is such a monomial as well, and the number $p^{\prime}$ (resp. $q^{\prime}, m$ ) for $\tau \otimes \varsigma$ is the sum of numbers $p^{\prime}$ (resp. $q^{\prime}, m$ ) for $\tau$ and $\sigma$. Therefore in this case the claim readily follows from the definitions (3.6.5), (3.6.6).

Consider the general case. Then by (3.1.2) we have $\tau=\sum_{i} \tau_{i}, \varsigma=\sum_{j} \varsigma_{j}$ where each $\tau_{i}$ and $\varsigma_{j}$ is a monomial in $v$. From (3.6.6) we obtain $\varrho\left(b_{l} B_{l}, \tau\right)_{V, v}=$ $\min _{i} \varrho\left(b_{l} B_{l}, \tau_{i}\right)_{V, v}, \varrho\left(b_{l} B_{l}, \varsigma\right)_{V, v}=\min _{j} \varrho\left(b_{l} B_{l}, \varsigma_{j}\right)_{V, v}$. Since $\tau \otimes \varsigma=\sum_{i, j} \tau_{i} \otimes \varsigma_{j}$ and each $\tau_{i} \otimes \varsigma_{j}$ is a monomial in $v$, we have

$$
\begin{aligned}
& \varrho\left(b_{l} B_{l}, \tau \otimes \varrho\right)_{V, v}=\min _{i, j} \varrho\left(b_{l} B_{l}, \tau_{i} \otimes \varrho_{j}\right)_{V, v}=\min _{i, j}\left(\varrho\left(b_{l} B_{l}, \tau_{i}\right)_{V, v}+\varrho\left(b_{l} B_{l}, \varsigma_{j}\right)_{V, v}\right) \\
& =\min _{i} \varrho\left(b_{l} B_{l}, \tau_{i}\right)_{V, v}+\min _{j} \varrho\left(b_{l} B_{l}, \varrho_{j}\right)_{V, v}=\varrho\left(b_{l} B_{l}, \tau\right)_{V, v}+\varrho\left(b_{l} B_{l}, \varrho\right)_{V, v} .
\end{aligned}
$$

Lemma 3.6.2. $\varrho\left(b_{l} B_{l}, \tau\right)_{V, v}$ depends only on $\tau$ and $b_{l} B_{l}$ but not on the choice of $V$ and $v_{1}, \ldots, v_{n}$.

Proof. First we remark that if $V^{\prime}$ is an open subset of $V$ and $\operatorname{supp}\left(B_{l}\right) \cap V^{\prime} \neq \varnothing$, then clearly $\varrho\left(b_{l} B_{l}, \tau\right)_{V^{\prime}, v}=\varrho\left(b_{l} B_{l}, \tau\right)_{V, v}$.

Now consider another open subset $\tilde{V}$ of $Y_{\text {sm }}$, and a system $\tilde{v}$ of functions $\tilde{v}_{1}, \ldots, \tilde{v}_{n} \in k[\tilde{V}]$ such that $\operatorname{supp}\left(B_{l}\right) \cap \tilde{V} \neq \varnothing$, the ideal of $\operatorname{supp}\left(B_{l}\right) \cap \tilde{V}$ in $k[\tilde{V}]$ is generated by $\tilde{v}_{n}$, and $d \tilde{v}_{1}(a), \ldots, d \tilde{v}_{n}(a)$ is a basis of $T_{a, \tilde{V}}^{*}$ for each point $a \in \tilde{V}$. We have to show that $\varrho\left(b_{l} B_{l}, \tau\right)_{V, v}=\varrho\left(b_{l} B_{l}, \tau\right)_{\tilde{V}, \tilde{v}}$. Since $\operatorname{supp}\left(B_{l}\right)$ is irreducible, $\operatorname{supp}\left(B_{l}\right) \cap V \cap \tilde{V} \neq \varnothing$. Hence using the remark above and replacing $V$ and $\tilde{V}$ by $V \cap \tilde{V}$ we can (and shall) assume that $V=\tilde{V}$. This reduces the problem to proving that

$$
\varrho\left(b_{l} B_{l}, \tau\right)_{V, v} \leqslant \varrho\left(b_{l} B_{l}, \tau\right)_{V, \tilde{v}}
$$

To show that (3.6.9) holds, we need the divisibility properties of some functions. Namely, the defining property of $\tilde{v}_{1}, \ldots, \tilde{v}_{n}$ implies that there are unique decompositions

$$
d v_{i}=\sum_{j=1}^{n} g_{i j} d \tilde{v}_{j}, \frac{\partial}{\partial v_{i}}=\sum_{j=1}^{n} h_{i j} \frac{\partial}{\partial \tilde{v}_{j}}, \quad \text { where } g_{i j}, h_{i j} \in k[V] .
$$


Since either of $v_{n}$ and $\tilde{v}_{n}$ is a generator of the ideal of $\operatorname{supp}\left(B_{l}\right) \cap V$ in $k[V]$, we have

$$
v_{n}=f \tilde{v}_{n}, \text { where } f \text { and } 1 / f \in k[V] .
$$

We claim that

$$
\tilde{v}_{n} \text { divides } g_{n i} \text { and } h_{i n} \text { in } k[V] \text { for all } i=1, \ldots, n-1 \text {. }
$$

To prove this, take a point $a \in \operatorname{supp}\left(B_{l}\right) \cap V$ and an index $i, 1 \leqslant i \leqslant n-1$. As $\tilde{v}_{n}(a)=0$, from (3.6.11) we deduce that

$$
d v_{n}(a)=f(a) d \tilde{v}_{n}(a)
$$

On the other hand, by (3.6.10), we have $d v_{n}(a)=\sum_{j=1}^{n} g_{n j}(a) d \tilde{v}_{j}(a)$. Since $d \tilde{v}_{1}(a), \ldots, d \tilde{v}_{n}(a)$ is a basis of $T_{a, V}^{*}$, the latter equality and (3.6.13) imply that $g_{n i}$ vanishes on $\operatorname{supp}\left(B_{l}\right) \cap V$, whence it is divisible by $\tilde{v}_{n}$ in $k[V]$. Further, by (3.1.1), the value of $d v_{n}(a)$ on $\frac{\partial}{\partial v_{i}}(a)$ is equal to 0 . On the other hand, by (3.6.13) and (3.6.10), it is equal to the value of $f(a) d \tilde{v}_{n}(a)$ on $\sum_{j=1}^{n} h_{i j}(a) \frac{\partial}{\partial \tilde{v}_{j}}(a)$ that in turn is equal to $f(a) h_{i n}(a)$. By (3.6.11), this implies that $h_{i n}(a)=0$. Thus $h_{\text {in }}$ vanishes on $\operatorname{supp}\left(B_{l}\right) \cap V$, whence it is divisible by $\tilde{v}_{n}$ in $k[V]$. This completes the proof of (3.6.12).

Now we can proceed to proving (3.6.9). By (3.6.6), for each nonzero $\delta_{j_{1} \ldots j_{q}}^{i_{1} \ldots i_{p}}$ we have $\varrho\left(b_{l} B_{l}, \delta_{j_{1} \ldots j_{q}}^{i_{1} \ldots i_{p}}\right)_{V, v}=\mu\left(b_{l} B_{l}, \delta_{j_{1} \ldots j_{q}}^{i_{1} \ldots i_{p}}\right)_{V, v}$. This and (3.6.7) imply that it suffices to prove (3.6.9) for the case when $\tau$ is a monomial in $v$,

$$
\tau=\frac{\partial}{\partial v_{i_{1}}} \otimes \cdots \otimes \frac{\partial}{\partial v_{i_{p}}} \otimes d v_{j_{1}} \otimes \cdots \otimes d v_{j_{q}}
$$

So assume that (3.6.14) holds. Then we prove (3.6.9) by induction with respect to the valency $p+q$. First we prove (3.6.9) for $p+q=1$, i.e., when $\tau=\frac{\partial}{\partial v_{i}}$ or $\tau=d v_{i}$ for some $i$. Use the notation of (3.6.5) for $\delta_{j_{1} \ldots j_{q}}^{i_{1} \ldots i_{p}}=\frac{\partial}{\partial v_{i}}$. We have $m=0, q^{\prime}=0$. If $i<n$, then $p^{\prime}=0$. If $i=n$, then $p^{\prime}=1$. So, by $(3.6 .5),(3.6 .6)$,

$$
\varrho\left(b_{l} B_{l}, \frac{\partial}{\partial v_{i}}\right)_{V, v}=\mu\left(b_{l} B_{l}, \frac{\partial}{\partial v_{i}}\right)_{V, v}= \begin{cases}0 & \text { if } i<n, \\ 1-b_{l} & \text { if } i=n .\end{cases}
$$

Similarly, using the notation of (3.6.5) for $\delta_{j_{1} \ldots j_{q}}^{i_{1} \ldots i_{p}}=d v_{i}$, we have: $m=0$, $p^{\prime}=0$; if $i<n$, then $q^{\prime}=0$, if $i=n$, then $q^{\prime}=1$. By (3.6.5), (3.6.6), this yields

$$
\varrho\left(b_{l} B_{l}, d v_{i}\right)_{V, v}=\mu\left(b_{l} B_{l}, d v_{i}\right)_{V, v}= \begin{cases}0 & \text { if } i<n \\ b_{l}-1 & \text { if } i=n .\end{cases}
$$


According to (3.6.10), (3.6.6) we have

$$
\begin{aligned}
& \varrho\left(b_{l} B_{l}, \frac{\partial}{\partial v_{i}}\right)_{V, \tilde{v}}=\min _{j} \mu\left(b_{l} B_{l}, h_{i j} \frac{\partial}{\partial \tilde{v}_{j}}\right)_{V, \tilde{v}}, \\
& \varrho\left(b_{l} B_{l}, d v_{i}\right)_{V, \tilde{v}}=\min _{j} \mu\left(b_{l} B_{l}, g_{i j} d \tilde{v}_{j}\right)_{V, \tilde{v}} .
\end{aligned}
$$

¿From (3.6.7) and (3.6.15), (3.6.16) (where $v_{i}, v, i$ are replaced by $\tilde{v}_{j}, \tilde{v}, j$ resp.) we obtain

$$
\begin{aligned}
& \mu\left(b_{l} B_{l}, h_{i j} \frac{\partial}{\partial \tilde{v}_{j}}\right)_{V, \tilde{v}}= \begin{cases}b_{l} m_{B_{l}, \operatorname{div}\left(h_{i j}\right)} & \text { if } j<n, \\
b_{l} m_{B_{l}, \operatorname{div}\left(h_{i j}\right)}+1-b_{l} & \text { if } j=n,\end{cases} \\
& \mu\left(b_{l} B_{l}, g_{i j} d \tilde{v}_{j}\right)_{V, \tilde{v}}= \begin{cases}b_{l} m_{B_{l}, \operatorname{div}\left(g_{i j}\right)} & \text { if } j<n, \\
b_{l} m_{B_{l}, \operatorname{div}\left(g_{i j}\right)}+b_{l}-1 & \text { if } j=n .\end{cases}
\end{aligned}
$$

By (3.6.10), (3.6.11) we have $m_{B_{l}, \operatorname{div}\left(h_{i j}\right)} \geqslant 0, m_{B_{l}, \operatorname{div}\left(g_{i j}\right)} \geqslant 0$ for all $i, j$, and $m_{B_{l}, \operatorname{div}\left(h_{i n}\right)} \geqslant 1, m_{B_{l}, \operatorname{div}\left(g_{n i}\right)} \geqslant 1$ for all $i<n$. Hence (3.6.18) implies

$$
\begin{aligned}
& \mu\left(b_{l} B_{l}, h_{i j} \frac{\partial}{\partial \tilde{v}_{j}}\right)_{V, \tilde{v}} \geqslant \begin{cases}0 & \text { if } j<n, \\
1 & \text { if } j=n, i<n, \\
1-b_{l} & \text { if } j=n, i=n,\end{cases} \\
& \mu\left(b_{l} B_{l}, g_{i j} d \tilde{v}_{j}\right)_{V, \tilde{v}} \geqslant \begin{cases}0 & \text { if } j<n, i<n, \\
b_{l} & \text { if } j<n, i=n, \\
b_{l}-1 & \text { if } j=n .\end{cases}
\end{aligned}
$$

Since $b_{l} \geqslant 1$, it follows from (3.6.17), (3.6.19), (3.6.15), (3.6.16) that

$$
\begin{aligned}
& \varrho\left(b_{l} B_{l}, \frac{\partial}{\partial v_{i}}\right)_{V, \tilde{v}} \geqslant\left\{\begin{array}{ll}
0 & \text { if } i<n, \\
1-b_{l} & \text { if } i=n,
\end{array}\right\}=\varrho\left(b_{l} B_{l}, \frac{\partial}{\partial v_{i}}\right)_{V, v}, \\
& \varrho\left(b_{l} B_{l}, d v_{i}\right)_{V, \tilde{v}} \geqslant\left\{\begin{array}{ll}
0 & \text { if } i<n, \\
b_{l}-1 & \text { if } i=n .
\end{array}\right\}=\varrho\left(b_{l} B_{l}, d v_{i}\right)_{V, v},
\end{aligned}
$$

whence (3.6.9) for $p+q=1$.

Now assume that $p+q>1$. Then we can write $\tau=\tau^{\prime} \otimes \tau^{\prime \prime}$ where $\tau^{\prime}$ and $\tau^{\prime \prime}$ are the monomials in $v$ that have valencies $<p+q$. By Lemma 3.6.1,

$$
\begin{aligned}
& \varrho\left(b_{l} B_{l}, \tau\right)_{V, v}=\varrho\left(b_{l} B_{l}, \tau^{\prime}\right)_{V, v}+\varrho\left(b_{l} B_{l}, \tau^{\prime \prime}\right)_{V, v}, \\
& \varrho\left(b_{l} B_{l}, \tau\right)_{V, \tilde{v}}=\varrho\left(b_{l} B_{l}, \tau^{\prime}\right)_{V, \tilde{v}}+\varrho\left(b_{l} B_{l}, \tau^{\prime \prime}\right)_{V, \tilde{v}},
\end{aligned}
$$

and by the inductive hypothesis,

$$
\begin{aligned}
\varrho\left(b_{l} B_{l}, \tau^{\prime}\right)_{V, v} & \leqslant \varrho\left(b_{l} B_{l}, \tau^{\prime}\right)_{V, \tilde{v}}, \\
\varrho\left(b_{l} B_{l}, \tau^{\prime \prime}\right)_{V, v} & \leqslant \varrho\left(b_{l} B_{l}, \tau^{\prime \prime}\right)_{V, \tilde{v}} .
\end{aligned}
$$


Clearly (3.6.9) follows from (3.6.20), (3.6.21).

Given Lemma 3.6.2, we denote $\varrho\left(b_{l} B_{l}, \tau\right)_{V, v}$ by $\varrho\left(b_{l} B_{l}, \tau\right)$.

Now we define the $\operatorname{divisor}_{\operatorname{div}_{B}}(\tau)$ for a positive divisor $B$ given by (3.6.2) as follows. Let $C \in \operatorname{Div}(Y)$ be a prime divisor, then

$$
m_{C, \operatorname{div}_{B}(\tau)}:=\left\{\begin{array}{l}
\varrho\left(b_{l} B_{l}, \tau\right) \text { if } C=B_{l} \text { for some } l, \\
m_{C, \operatorname{div}(\tau)} \text { if } C \neq B_{l} \text { for all } l .
\end{array}\right.
$$

Note that (3.6.22), (3.2.1), (3.2.2) and Lemma 3.6.1 imply

Corollary 3.6.1. For any positive divisor $B$ and nonzero tensor fields $\tau \in$ $\Gamma_{\mathrm{rat}}\left(T_{q}^{p}(Y)_{\mathrm{sm}}\right), \varsigma \in \Gamma_{\mathrm{rat}}\left(T_{t}^{s}(Y)_{\mathrm{sm}}\right)$ the following equality holds:

$$
\operatorname{div}_{B}(\tau \otimes \varsigma)=\operatorname{div}_{B}(\tau)+\operatorname{div}_{B}(\varsigma) .
$$

3.7. Behavior at étale morphisms. Let $\alpha: Z \rightarrow Y$ be a surjective étale morphism of irreducible smooth algebraic varieties. Let $S$ be a subvariety of codimension 1 in $Y$, and let $f$ be a local equation of $S$ at a point $y \in S_{\mathrm{sm}}$. Then $d f(y) \neq 0$, and as $\alpha$ is étale, $d\left(\alpha^{*}(f)\right)(z) \neq 0$ for any point $z \in \alpha^{-1}(y)$. This implies that $\alpha^{*}(f)$ is a local equation at $z$ of the subvariety $\alpha^{-1}(S)$ of $Z$. Hence if $C \in \operatorname{Div}(Y)$ and $C^{\prime} \in \operatorname{Div}(Z)$ are prime divisors, then $m_{C^{\prime}, \alpha^{*}(C)}=0$ or 1 , depending on whether $\operatorname{supp}\left(C^{\prime}\right)$ is an irreducible component of $\alpha^{-1}(\operatorname{supp}(C))$ or not.

Lemma 3.7.1. Let $\tau$ be a nonzero rational tensor field on $Y$ and let $B \in$ $\operatorname{Div}(Y)$ be a positive divisor. If $C \in \operatorname{Div}(Y)$ and $C^{\prime} \in \operatorname{Div}(Z)$ are prime divisors such that $m_{C^{\prime}, \alpha^{*}(C)}=1$, then

$$
m_{C, \operatorname{div}_{B}(\tau)}=m_{C^{\prime}, \operatorname{div}_{\alpha^{*}(B)}\left(\alpha^{*}(\tau)\right)} \cdot
$$

Proof. We use the notation of Subsections 3.1, 3.2 and 3.3, so that (3.1.2) and (3.3.5) hold, and $t$ is a local equation of $C$. Then $\alpha^{*}(t)$ is a local equation of $C^{\prime}$ in an appropriate neighborhood of a point of $\operatorname{supp}\left(C^{\prime}\right)$. Hence

$$
m_{C, \operatorname{div}\left(\tau_{j_{1} \ldots j_{q}}^{i_{1} \ldots i_{p}}\right)}=m_{C^{\prime}, \operatorname{div}\left(\alpha^{*}\left(\tau_{j_{1} \ldots j_{q}}^{i_{1} \ldots i_{p}}\right)\right)}
$$

for all $i_{1}, \ldots, i_{p}, j_{1}, \ldots, j_{q}$. Then from (3.1.2), (3.3.4), (3.3.5), (3.2.1), (3.2.2) and (3.7.2) we deduce that

$$
m_{C, \operatorname{div}(\tau)}=m_{C^{\prime}, \operatorname{div}\left(\alpha^{*}(\tau)\right)} \cdot
$$

From (3.7.3) and (3.6.22) it follows that (3.7.1) holds if $C \neq B_{i}$ for each $B_{i}$ in decomposition (3.6.2).

Now assume that $C=B_{l}$ for some $l$. Then $b_{l}=m_{C, B}=m_{C^{\prime}, \alpha^{*}(B)}$. Use the notation of Subsection 3.6. By (3.3.3), the local equations of $C$ and $C^{\prime}$ in the appropriate neighborhoods are respectively $v_{n}$ and $u_{n}$. Therefore from (3.1.2), (3.3.5), (3.6.5) and (3.6.6) we deduce the equality $\varrho\left(b_{l} C, \tau\right)=\varrho\left(b_{l} C^{\prime}, \alpha^{*}(\tau)\right)$. By (3.6.22), this yields (3.7.1). 
INVARIANT TENSOR FIELDS FOR ALGEBRAIC FINITE GROUP ACTIONS 19

Corollary 3.7.1. The following properties are equivalent:

(i) $\operatorname{div}_{B}(\tau) \geqslant 0$,

(ii) $\operatorname{div}_{\alpha^{*}(B)}\left(\alpha^{*}(\tau)\right) \geqslant 0$.

3.8. Regularity of the pull back of a tensor field on a quotient variety: General criterion. Let $X$ be an irreducible smooth algebraic variety endowed with a faithful action of a finite group $G$. Assume that there exists a geometric quotient (2.4.1). Recall that we defined by (2.6.2) the reflection divisor $R_{X / G}$ of $X / G$,

$$
R_{X / G}:=r_{1} R_{1}+\ldots+r_{d} R_{d} \in \operatorname{Div}(X / G) .
$$

The following theorem is the main result of Section 3.

Theorem 3.8.1. Let $X$ be an irreducible smooth algebraic variety endowed with an action of a finite group $G$. Assume that there exists a geometric quotient (2.4.1). Let $\tau$ be a nonzero rational tensor field on $X / G$. Then the following properties are equivalent:

(i) $\operatorname{div}\left(\pi_{X}^{*}(\tau)\right) \geqslant 0$, i.e., the pull back $\pi_{X}^{*}(\tau)$ of $\tau$ is regular on $X$;

(ii) $\operatorname{div}_{R_{X / G}}(\tau) \geqslant 0$.

Proof. We can (and shall) assume that the action is faithful. By the definitions of $\operatorname{div}\left(\pi_{X}^{*}(\tau)\right)$ and $\operatorname{div}_{R_{X / G}}(\tau)$, either of conditions (i) and (ii) implies that $\tau$ is regular on $(X / G)_{0}$ and $\pi_{X}^{*}(\tau)$ is regular on $X_{0}$. So we can (and shall) assume that $\tau$ and $\pi_{X}^{*}(\tau)$ share these properties.

If $R_{X / G}=0$, the claim follows from (2.6.3) and Corollary 3.5.1. So we shall assume that $R_{X / G}>0$, i.e., $(X / G)_{1} \neq \varnothing$. As $\operatorname{dom}\left(\pi_{X}^{*}(\tau)\right) \supseteq X_{0}$, regularity of $\pi_{X}^{*}(\tau)$ on $X$ is equivalent to regularity of $\pi_{X}^{*}(\tau)$ on $X_{1}$ (see (3.1.5)-(3.1.6)).

Let $x$ be a point of $X_{1}$ and $z:=\pi_{X}(x) \in(X / G)_{1}$. By (2.6.4), there is a unique irreducible component $(X / G)_{1}^{l}$ of $(X / G)_{1}$ (see $\left.(2.6 .1)\right)$ containing $z$. As $\left.\pi_{X}\right|_{W}: \pi_{X}^{-1}(W) \rightarrow W$ for any open subset $W$ of $X / G$ is the geometrical quotient for the action of $G$ on $\pi_{X}^{-1}(W)$, cf. [PV, 4.2], the problem is local, i.e., we have to prove that $\pi_{X}^{*}(\tau)$ is regular at $x$ if and only if $\varrho\left(r_{l} R_{l}, \tau\right) \geqslant 0$, see (3.6.22), (2.6.2). To that end we apply Lemma 2.5.1.

Let $U$ be the $G_{x}$-stable neighborhood of $x$ from the proof of this lemma such that the commutative diagram (2.5.2) holds. Then we have

$$
\begin{gathered}
\left.\pi_{X}^{*}(\tau)\right|_{U}=\left(\left(\sigma_{S} / G\right) \circ \pi_{U, G_{x}}\right)^{*}(\tau)=\pi_{U, G_{x}}^{*}(\theta), \\
\text { where } \theta=\left(\sigma_{S} / G\right)^{*}(\tau)=\left(\pi_{U, G_{x}}\right)_{*}\left(\pi_{X}^{*}(\tau)\right) .
\end{gathered}
$$

Let $D \in \operatorname{Div}\left(U / G_{x}\right)$ be the prime divisor such that $\operatorname{supp}(D)$ is the irreducible component of $\left(\sigma_{S} / G\right)^{-1}\left((X / G)_{1}^{l}\right)$ containing $y=\pi_{X, G_{x}}(x)$. Since $\sigma_{S} / G$ is étale, we have $m_{D,\left(\sigma_{S} / G\right)^{*}\left(R_{X / G}\right)}=r_{l}$, and by Lemma 3.7.1, our problem is reduced to proving that the following properties are equivalent:

(a) $\left.\pi_{X}^{*}(\tau)\right|_{U}$ is regular at $x$,

(b) $\varrho\left(r_{l} D, \theta\right) \geqslant 0$. 
Let $u_{1}, \ldots, u_{n}$ and $v_{1}, \ldots v_{n}$ be the local parameters from Lemma 2.5.1. Replacing, if necessary, $U$ by a smaller neighborhood $U^{\prime}$ of $x$, and then $U^{\prime}$ by $\cap_{g \in G_{x}} g \cdot U^{\prime}$, we can (and shall) assume that

○ $U=\pi_{X, G_{x}}^{-1}(V)$ where $V$ is a neighborhood of $y$ in $X / G_{x}$,

- $X_{1} \cap U$ is irreducible,

$\circ u_{1}, \ldots, u_{n} \in k[U]$,

$\circ v_{1}, \ldots, v_{n} \in k[V]$

○ the ideal of $X_{1} \cap U$ in $k[U]$ is generated by $u_{n}$,

○ the ideal of $\pi_{U, G_{x}}\left(X_{1}\right)$ in $k[V]$ is generated by $v_{n}$,

- $d u_{1}(a), \ldots, d u_{n}(a)$, is a basis of $T_{a, U}^{*}$ for each $a \in U$,

○ $d v_{1}(b), \ldots, d v_{n}(b)$ is a basis of $T_{b, V}^{*}$ for each $b \in V$.

¿From (2.5.1) and (3.1.1) we deduce that

(3.8.2) $\pi_{X, G_{x}}^{*}\left(d v_{i}\right)=d u_{i}$ for $1 \leqslant i \leqslant n-1$, and $\pi_{X, G_{x}}^{*}\left(d v_{n}\right)=r_{l} u_{n}^{r_{l}-1} d u_{n}$, (3.8.3) $\pi_{X, G_{x}}^{*}\left(\frac{\partial}{\partial v_{i}}\right)=\frac{\partial}{\partial u_{i}}$ for $1 \leqslant i \leqslant n-1$, and $\pi_{X, G_{x}}^{*}\left(\frac{\partial}{\partial v_{n}}\right)=\frac{1}{r_{l} u_{n}^{r_{l}-1}} \frac{\partial}{\partial u_{n}}$.

By Lemma 2.5.1 and $d u_{i}\left(\frac{\partial}{\partial u_{j}}\right)=\delta_{i j}$, for each $g \in G_{x}$ we have

$$
\begin{gathered}
g \cdot d u_{1}=d u_{1}, \ldots, g \cdot d u_{n-1}=d u_{n-1}, g \cdot d u_{n}=\chi(g) d u_{n}, \\
g \cdot \frac{\partial}{\partial u_{1}}=\frac{\partial}{\partial u_{1}}, \ldots, \quad g \cdot \frac{\partial}{\partial u_{n-1}}=\frac{\partial}{\partial u_{n-1}}, \quad g \cdot \frac{\partial}{\partial u_{n}}=\chi(g)^{-1} \frac{\partial}{\partial u_{n}} .
\end{gathered}
$$

In view of the properties of $u_{1}, \ldots, u_{n}$, there is a unique decomposition

$$
\pi_{X}^{*}(\tau)=\sum_{i_{1}, \ldots, i_{p}, j_{1}, \ldots, j_{q}} \psi_{j_{1} \ldots j_{q}}^{i_{1} \ldots i_{p}} \frac{\partial}{\partial u_{i_{1}}} \otimes \cdots \otimes \frac{\partial}{\partial u_{i_{p}}} \otimes d u_{j_{1}} \otimes \cdots \otimes d u_{j_{q}}
$$

where $\psi_{j_{1} \ldots j_{q}}^{i_{1} \ldots i_{p}} \in k(U)$.

Since $\pi_{X}^{*}(\tau)$ is $G_{x}$-invariant, (3.8.4) and the uniqueness of decomposition (3.8.5) imply that each summand in the right-hand side of (3.8.2) is $G_{x^{-}}$invariant.

Consider such a summand

$$
\left(\pi_{X}^{*}(\tau)\right)_{j_{1} \ldots j_{q}}^{i_{1} \ldots i_{p}}:=\psi_{j_{1} \ldots j_{q}}^{i_{1} \ldots i_{p}} \frac{\partial}{\partial u_{i_{1}}} \otimes \cdots \otimes \frac{\partial}{\partial u_{i_{p}}} \otimes d u_{j_{1}} \otimes \cdots \otimes d u_{j_{q}}
$$

and assume that in (3.8.6) there are exactly $p^{\prime}$ factors $\frac{\partial}{\partial u_{n}}$ and exactly $q^{\prime}$ factors $d u_{n}$. Divide $p^{\prime}-q^{\prime}$ by $r_{l}:=\left|G_{x}\right|$ (see (2.6.2)) with reminder:

$$
p^{\prime}-q^{\prime}=s r_{l}+t, s, t \in \mathbb{Z}, 0 \leqslant t<r_{l} \text {. }
$$

Since the order of $\chi$ is $\left|G_{x}\right|$, and the field (3.8.6) is $G_{x}$-invariant, (3.8.4) and (3.8.7) yield

$$
g \cdot \psi_{j_{1} \ldots j_{q}}^{i_{1} \ldots i_{p}}=\chi(g)^{t} \psi_{j_{1} \ldots j_{q}}^{i_{1} \ldots i_{p}} \quad \text { for all } g \in G_{x}
$$


(a) $\Rightarrow(\mathrm{b})$. Assume that $\left.\pi_{X}^{*}(\tau)\right|_{U}$ is regular at $x$. Shrinking $U$ if necessary we can (and shall) assume that each $\psi_{j_{1} \ldots j_{q}}^{i_{1} \ldots i_{p}}$ is contained in $k[U]$. We claim that

$$
\psi_{j_{1} \ldots j_{q}}^{i_{1} \ldots i_{p}}=u_{n}^{t} \tilde{\psi}_{j_{1} \ldots j_{q}}^{i_{1} \ldots i_{p}} \text { for some } \tilde{\psi}_{j_{1} \ldots j_{q}}^{i_{1} \ldots i_{p}} \in k[U]^{G_{x}}
$$

Indeed, if $t=0$, then $\psi_{j_{1} \ldots j_{q}}^{i_{1} \ldots i_{p}} \in k[U]^{G_{x}}$ by (3.8.8), so (3.8.9) holds. Let $t>0$. As $t<\left|G_{x}\right|$, there is $g_{0} \in G_{x}$ such that $\chi\left(g_{0}\right)^{t} \neq 1$. Take a point $a \in X_{1} \cap U$. Since $a \in X^{G_{x}}$, from (3.8.9) we obtain that $g \cdot \psi_{j_{1} \ldots j_{q}}^{i_{1} \ldots i_{p}}(a)=$ $\psi_{j_{1} \ldots j_{q}}^{i_{1} \ldots i_{p}}\left(g^{-1} \cdot a\right)=\psi_{j_{1} \ldots j_{q}}^{i_{1} \ldots i_{p}}(a)=\chi\left(g_{0}\right)^{t} \psi_{j_{1} \ldots j_{q}}^{i_{1} \ldots i_{p}}(a)$, whence $\psi_{j_{1} \ldots j_{q}}^{i_{1} \ldots i_{p}}(a)=0$. Thus $\psi_{j_{1} \ldots j_{q}}^{i_{1} \ldots i_{p}}$ vanishes on $X_{1} \cap U$. Hence $\psi_{j_{1} \ldots j_{q}}^{i_{1} \ldots i_{p}}=u_{n} \tilde{\psi}_{j_{1} \ldots j_{q}}^{i_{1} \ldots i_{p}}$ for some $\tilde{\psi}_{j_{1} \ldots j_{q}}^{i_{1} \ldots i_{p}} \in k[U]$. As $k[U]$ is an integral domain, from this, (3.8.9) and Lemma 2.5.1 we deduce that $g \cdot \tilde{\psi}_{j_{1} \ldots j_{q}}^{i_{1} \ldots i_{p}}=\chi(g)^{t-1} \tilde{\psi}_{j_{1} \ldots j_{q}}^{i_{1} \ldots i_{p}}$ for all $g \in G_{x}$. Now the same arguments can be applied to $\tilde{\psi}_{j_{1} \ldots j_{q}}^{i_{1} \ldots i_{p}}$, and so on. Whence the claim.

Now, as $\tilde{\psi}_{j_{1} \ldots j_{q}}^{i_{1} \ldots i_{p}} \in k[U]^{G_{x}}$, we have $\tilde{\psi}_{j_{1} \ldots j_{q}}^{i_{1} \ldots i_{p}}=\pi_{X, G_{x}}^{*}\left(\varepsilon_{j_{1} \ldots j_{q}}^{i_{1} \ldots i_{p}}\right)$ for some

$$
\varepsilon_{j_{1} \ldots j_{q}}^{i_{1} \ldots i_{p}} \in k[V]
$$

Hence using (3.8.2), (3.8.3) and (3.8.9), we can rewrite the field (3.8.6) as follows

$$
\left(\pi_{X}^{*}(\tau)\right)_{j_{1} \ldots j_{q}}^{i_{1} \ldots i_{p}}=\pi_{X, G_{x}}^{*}\left(r_{l}^{p^{\prime}-q^{\prime}} v_{n}^{p^{\prime}-q^{\prime}-s} \varepsilon_{j_{1} \ldots j_{q}}^{i_{1} \ldots i_{p}} \frac{\partial}{\partial v_{i_{1}}} \otimes \cdots \otimes \frac{\partial}{\partial v_{i_{p}}} \otimes d v_{j_{1}} \otimes \cdots \otimes d v_{j_{q}}\right) .
$$

This equality and (3.8.5), (3.8.6), (3.8.1) imply that

$$
\begin{gathered}
\theta=\sum_{i_{1}, \ldots, i_{p}, j_{1}, \ldots j_{q}}\left(\pi_{U, G_{x}}\right)_{*}\left(\pi_{X}^{*}(\tau)\right)_{j_{1} \ldots j_{q}}^{i_{1} \ldots i_{p}} \\
=\sum_{i_{1}, \ldots, i_{p}, j_{1}, \ldots j_{q}} \theta_{j_{1} \ldots j_{q}}^{i_{1} \ldots i_{p}} \frac{\partial}{\partial v_{i_{1}}} \otimes \cdots \otimes \frac{\partial}{\partial v_{i_{p}}} \otimes d v_{j_{1}} \otimes \cdots \otimes d v_{j_{q}}
\end{gathered}
$$

where

$$
\theta_{j_{1} \ldots j_{q}}^{i_{1} \ldots i_{p}}=r_{l}^{p^{\prime}-q^{\prime}} v_{n}^{p^{\prime}-q^{\prime}-s} \varepsilon_{j_{1} \ldots j_{q}}^{i_{1} \ldots i_{p}}
$$

Since $v_{n}$ is the local equation of $D$ at $y$, it follows from (3.8.12) and (3.8.10) that

$$
m_{D, \operatorname{div}\left(\theta_{j_{1} \ldots j_{q}}^{i_{1} \ldots i_{p}}\right)}-p^{\prime}+q^{\prime}+s \geqslant 0 .
$$

Taking into account the identity

$$
\begin{aligned}
\left(r_{l}-1\right)\left(q^{\prime}-p^{\prime}\right) & +r_{l} m_{D, \operatorname{div}\left(\theta_{j_{1} \ldots j_{q}}^{i_{1} \ldots i_{p}}\right)} \\
& =r_{l}\left(m_{D, \operatorname{div}\left(\theta_{j_{1} \ldots j_{q}}^{i_{1} \ldots p_{p}}\right)}-p^{\prime}+q^{\prime}+s\right)+t
\end{aligned}
$$


we deduce from $(3.8 .13),(3.8 .7)$ and $r_{l}>0$ that

$$
\left(r_{l}-1\right)\left(q^{\prime}-p^{\prime}\right)+r_{l} m_{D, \operatorname{div}\left(\theta_{j_{1} \ldots j_{q}}^{i_{1} \ldots i_{p}}\right)} \geqslant 0 .
$$

Now (3.8.11), (3.8.14) and definitions (3.6.5), (3.6.6) immediately imply that $\varrho\left(r_{l} D, \theta\right) \geqslant 0$.

$(\mathrm{b}) \Rightarrow(\mathrm{a})$. Assume that $\varrho\left(r_{l} D, \theta\right) \geqslant 0$. In view of the properties of $v_{1}, \ldots, v_{n}$, there is a unique decomposition

$$
\begin{gathered}
\theta=\sum_{i_{1}, \ldots, i_{p}, j_{1}, \ldots, j_{q}} \vartheta_{j_{1} \ldots j_{q}}^{i_{1} \ldots i_{p}}, \quad \text { where } \\
\vartheta_{j_{1} \ldots j_{q}}^{i_{1} \ldots i_{p}}=\theta_{j_{1} \ldots j_{q}}^{i_{1} \ldots i_{p}} \frac{\partial}{\partial v_{i_{1}}} \otimes \cdots \otimes \frac{\partial}{\partial v_{i_{p}}} \otimes d v_{j_{1}} \otimes \cdots \otimes d v_{j_{q}}
\end{gathered}
$$

for some $\theta_{j_{1} \ldots j_{q}}^{i_{1} \ldots i_{p}} \in k(V)$.

Assume that in (3.8.17) there are exactly $p^{\prime}$ factors $\frac{\partial}{\partial v_{n}}$ and exactly $q^{\prime}$ factors $d v_{n}$. Let $s$ and $t$ be defined by (3.8.7). As $v_{n}$ is a local equation of $D$ at $y$, the definition of $\varrho\left(r_{l} D, \theta\right)$ (see (3.6.5), (3.6.6)) and the inequality $\varrho\left(r_{l} D, \theta\right) \geqslant 0$ imply that (3.8.15) holds. ¿From here, (3.8.14), (3.8.7) and $r_{l}>0$ we deduce that (3.8.13) holds. In turn this implies that shrinking $V$ if necessary, we can (and shall) assume that

$$
\theta_{j_{1} \ldots j_{q}}^{i_{1} \ldots i_{p}}=v_{n}^{p^{\prime}-q^{\prime}-s} \tilde{\theta}_{j_{1} \ldots j_{q}}^{i_{1} \ldots i_{p}}, \quad \text { where } \tilde{\lambda}_{j_{1} \ldots j_{q}}^{i_{1} \ldots i_{p}} \in k[V] .
$$

Then (3.8.17), (3.8.18), (3.8.2), (3.8.3), (2.5.1) yield

$$
\pi_{U, G_{x}}^{*}\left(\vartheta_{j_{1} \ldots j_{q}}^{i_{1} \ldots i_{p}}\right)=r_{l}^{q^{\prime}-p^{\prime}} u_{n}^{t} \pi_{U, G_{x}}^{*}\left(\tilde{\theta}_{j_{1} \ldots j_{q}}^{i_{1} \ldots i_{p}}\right) \frac{\partial}{\partial u_{i_{1}}} \otimes \cdots \otimes \frac{\partial}{\partial u_{i_{p}}} \otimes d u_{j_{1}} \otimes \cdots \otimes d u_{j_{q}} .
$$

As $t \geqslant 0$ and $\tilde{\theta}_{j_{1} \ldots j_{q}}^{i_{1} \ldots i_{p}} \in k[V]$, this shows that $\pi_{U, G_{x}}^{*}\left(\vartheta_{j_{1} \ldots j_{q}}^{i_{1} \ldots i_{p}}\right)$ is regular on $U$. Hence by (3.8.16) and (3.8.1), the field $\left.\pi_{X}^{*}(\tau)\right|_{U}$ is regular on $U$.

\section{Some Applications}

4.1. Generalization of Solomon's theorem. Let $V$ be a finite dimensional vector space over $k$ and $G$ a finite subgroup of $\mathrm{GL}(V)$. It is well known that the following properties are equivalent, e.g., see $[\mathrm{PV}]$ :

(i) $V / G$ is smooth.

(ii) $V / G$ is isomorphic to affine space.

(iii) $G$ is generated by pseudo-reflections.

Assume that conditions (i)-(iii) hold. Then Solomon's theorem can be reformulated as the statement that the algebra of all invariant regular differential forms on $V$ is the pull back of the algebra of all regular differential forms on $V / G$.

In $[\mathrm{M}]$ this statement was generalized to proper smooth polar actions of Lie groups on Riemannian manifolds, and in [B] to algebraic actions of reductive groups on smooth affine algebraic varieties with smooth categorical quotients. From Theorem 3.8.1 we deduce the following 
INVARIANT TENSOR FIELDS FOR ALGEBRAIC FINITE GROUP ACTIONS 23

Corollary 4.1.1. Let $X$ be an irreducible smooth algebraic variety endowed with an action of a finite group $G$. Assume that there exists a geometric quotient (2.4.1). Let $\tau$ be a rational differential form on $X / G$. Then $\pi_{X}^{*}(\tau)$ is regular on $X$ if and only if $\tau$ is regular on $(X / G)_{\mathrm{sm}}$.

Proof. By Theorem 3.8.1, we have to show that $\operatorname{dom}(\tau)=(X / G)_{\mathrm{sm}}$ is equivalent to $\operatorname{div}_{R_{X / G}}(\tau) \geqslant 0$. As either of these conditions implies $\operatorname{dom}(\tau) \supseteq(X / G)_{0}$, we can (and shall) assume that this inclusion holds. As $(X / G)_{1} \subset(X / G)_{\mathrm{sm}}$ by Lemma 2.5.1, it follows from $(3.1 .5),(3.1 .6)$ that $\operatorname{dom}(\tau)=(X / G)_{\mathrm{sm}}$ is equivalent to $\operatorname{dom}(\tau) \supseteq(X / G)_{1}$.

We can (and shall) assume that the action is faithful. We use the notation of Subsection 3.6 for $Y=X$ and $B=R_{X / G}$. The problem is local, i.e., we have to show that $\operatorname{dom}(\tau) \supseteq(X / G)_{1}^{l}$ (see (2.6.1)) iff $\varrho\left(r_{l} R_{l}, \tau\right) \geqslant 0$. For each field (3.6.4) we have $p^{\prime}=p=0$ and $q^{\prime} \leqslant 1$ by the skew symmetry condition. Whence $p^{\prime}-q^{\prime}=0$ or -1 . In both cases $p^{\prime}-q^{\prime}-s=0$. Hence in our case $m-p^{\prime}+q^{\prime}+s \geqslant 0$ is equivalent to $m \geqslant 0$, and we are done.

4.2. Rational tensor fields of type $\left(\begin{array}{l}\otimes p \\ \wedge q\end{array}\right)$. Consider a more general type of rational tensor fields than differential forms. Namely, let $Y$ be an irreducible algebraic variety. Consider the vector bundle $T\left(Y_{\mathrm{sm}}\right)^{\otimes p} \otimes \wedge^{q} T^{*}\left(Y_{\mathrm{sm}}\right)$ over $Y_{\mathrm{sm}}$. Its rational sections are precisely rational tensor fields of type $\left(\begin{array}{l}p \\ q\end{array}\right)$ on $Y$ that are skew symmetric with respect to the covariant entries. We call them rational tensor fields of type $\left(\begin{array}{l}\otimes p \\ \wedge q\end{array}\right)$. It is clear that pull backs and push forwards of rational tensor fields of type $\left(\begin{array}{l}\otimes p \\ \wedge q\end{array}\right)$ are rational tensor field of type $\left(\begin{array}{l}\otimes p \\ \wedge q\end{array}\right)$ as well.

Corollary 4.2.1. Let $X$ be an irreducible smooth algebraic variety endowed with an action of a finite group $G$. Assume that there exists a geometric quotient (2.4.1). Let $\varphi$ be a G-invariant regular tensor field of type $\left(\begin{array}{l}\otimes p \\ \wedge q\end{array}\right)$ on $X$. Then $\pi_{X *}(\varphi)$ is regular on $(X / G)_{\mathrm{sm}}$.

Proof. We have $\varphi=\pi_{X}^{*}(\tau)$ for $\tau:=\pi_{X *}(\varphi)$. Arguing like in the proof of Corollary 4.1.1 we reduce the problem to showing that $\varrho\left(r_{l} R_{l}, \tau\right) \geqslant 0$ implies $\operatorname{dom}(\tau) \supseteq(X / G)_{1}^{l}$. For each field (3.6.4) we have $q^{\prime} \leqslant 1$ by the skew symmetry condition. Hence $p^{\prime}-q^{\prime} \geqslant-1$. If $p^{\prime}-q^{\prime}=-1$, resp. $\geqslant 0$, then $s=-1$, resp. $\geqslant 0$. Therefore $p^{\prime}-q^{\prime}-s \geqslant 0$. Hence $m-p^{\prime}+q^{\prime}+s \geqslant \varrho\left(r_{l} R_{l}, \tau\right) \geqslant 0$ implies $m \geqslant 0$, and we are done.

4.3. Partially symmetric tensor fields. Let $Y$ be an irreducible algebraic variety. Rational sections of $\mathrm{S}^{q_{1}}\left(T^{*}\left(Y_{\mathrm{sm}}\right)\right) \otimes \cdots \otimes \mathrm{S}^{q_{d}}\left(T^{*}\left(Y_{\mathrm{sm}}\right)\right)$ are called $r a-$ tional multi-symmetric covariant tensor fields of type $\left(q_{1}, \ldots, q_{d}\right)$ on $Y$. If $Y$ is smooth and $\sigma$ is such a field, then

$$
T(\operatorname{dom}(\sigma))^{\oplus d} \rightarrow k, v \mapsto(\sigma(a))(v) \text { for each } a \in \operatorname{dom}(\sigma), v \in T_{a, Y}^{\oplus d}
$$

is a rational function on the algebraic variety $T(Y)^{\oplus d}$, homogeneous of multidegree $\left(q_{1}, \ldots, q_{d}\right)$ with respect to the natural diagonal action of $k^{\times}$on $T(Y)^{\oplus d}$. 
This function is regular on $T(Y)^{\oplus d}$ iff $\sigma$ is regular on $Y$. Every such rational function is obtained in this way. Therefore if $Y$ is smooth and endowed with an action of a finite group $G$, Theorem 3.8.1 provides a characterization of $G$-invariant regular functions on $T(Y)^{\oplus d}$ in terms of rational multi-symmetric covariant tensor fields on $Y / G$.

If $Y=V$ is a vector space and $G$ a subgroup of $\mathrm{GL}(V)$, we have $T(V)^{\oplus d}=$ $V^{\oplus(d+1)}$ and the action of $G$ on $T(V)^{\oplus d}$ coincides with the diagonal action on $V^{\oplus(d+1)}$. Thus Theorem 3.8.1 yields a characterization of $G$-invariant polynomials on $V^{\oplus(d+1)}$ in terms of rational multi-symmetric covariant tensor fields on $V / G$. Note that algebras $k[V \oplus \ldots \oplus V]^{G}$ were studied in $[\mathrm{Hu}]$ for some groups $G$ generated by pseudo-reflections (i.e., when $V / G$ is smooth).

\section{Lifting Automorphisms of Quotients}

5.1. Let $X$ be an irreducible algebraic variety endowed with an action of a finite group $G$ such that there exists the geometric quotients (2.4.1). If $\alpha$ is an automorphism of $X / G$ induced by a $G$-automorphism of $X$, then $\alpha\left((X / G)_{0}\right)=$ $(X / G)_{0}$ and $\alpha_{*}\left(R_{X / G}\right)=R_{X / G}$.

The following theorem is a conversion of this statement for linear actions. It can be seen as complementing the results in $[\mathrm{B}]$, [Sch], [Lo], and [KLM].

Theorem 5.1.1. Let $V$ be a finite dimensional complex vector space and let $G \subset \mathrm{GL}(V)$ be a finite group. Let $\psi$ be an automorphism of the algebraic variety $V / G$ such that $\psi(V / G)_{0} \subseteq(V / G)_{0}$ and $\psi_{*}\left(R_{V / G}\right)=R_{V / G}$. Then there is an automorphism $\varphi$ of the algebraic variety $V$ such that the following diagram is commutative

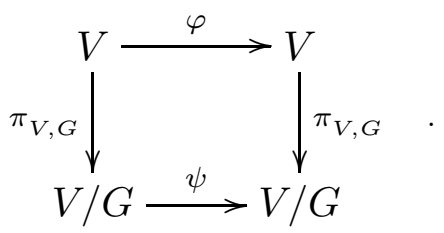

Proof. It is proved in $[\mathrm{KLM}]$ that there is an analytic automorphism $\varphi$ of the analytic space $V$ such that the diagram (5.1.1) is commutative. Hence the claim immediately follows from

Lemma 5.1.1. Let $X, Y$ and $Z$ be complex algebraic varieties. Assume that $Y$ is irreducible and smooth and $X$ is endowed with an action of a finite group $H$. Let the maps $\alpha, \beta, \gamma$ in the commutative diagram

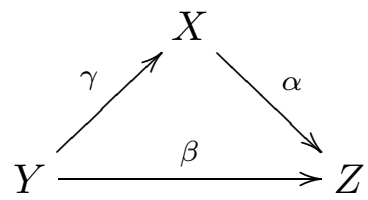


have the properties:

(i) $\alpha$ and $\beta$ are morphisms,

(ii) every nonempty fiber of $\alpha$ is an $H$-orbit,

(iii) $\gamma$ is analytic.

Then $\gamma$ is a morphism of algebraic varieties.

Proof. Consider in $X \times Y$ the subsets

$$
\begin{gathered}
\Delta=\{(x, y) \in X \times Y \mid \alpha(x)=\beta(y)\}, \\
\Gamma=\{(\gamma(y), y) \in X \times Y \mid y \in Y\} .
\end{gathered}
$$

Clearly $\Delta$ is Zariski closed. As $\Gamma$ is the graph of $\gamma$ and $Y$ is smooth, (iii) implies that $\Gamma$ is an analytic subset of $X \times Y$, cf. [He, Ch. IV, no. 5]; clearly it is analytically isomorphic to $Y$.

Consider the action of $H$ on $X \times Y$ through the first factor. By (ii), we have

$$
\Delta=\bigcup_{h \in H} h(\Gamma)
$$

Taking into account that the decompositions of any complex algebraic variety into irreducible components in the categories of algebraic varieties and analytic sets coincide, cf. [GR, Ch. V, B, Proposition 1], we deduce from irreducibility of $Y$ that $\Gamma$ is an irreducible analytic set. Hence (5.2.1) yields that each $h(\Gamma)$ is an irreducible component of the analytic set $\Delta$. Thereby $h(\Gamma)$ is an irreducible component of the algebraic variety $\Delta$. Thus $\Gamma$ is a Zariski closed subset of $X \times Y$.

Let $X \stackrel{\pi_{1}}{\longleftarrow} X \times Y \stackrel{\pi_{2}}{\longrightarrow} Y$ be the projections. As $\left.\pi_{2}\right|_{\Gamma}: \Gamma \rightarrow Y$ is a bijective morphism of algebraic varieties and $Y$ is normal, Zariski's Main Theorem implies that $\left.\pi_{2}\right|_{\Gamma}$ is an isomorphism of algebraic varieties. Therefore $\left(\left.\pi_{2}\right|_{\Gamma}\right)^{-1}$ is a morphism (actually, an isomorphism) of algebraic varieties. Whence $\gamma=\pi_{1} \circ\left(\left.\pi_{2}\right|_{\Gamma}\right)^{-1}$ is a morphism as well.

5.2. Remarks. By Lefschetz's principle, see, e.g., [Si, p. 164], Theorem 5.1.1 remains true over any algebraically closed field of characteristic 0 . Notice that the proof of the result from [KLM] used in the proof of Theorem 5.1.1 involves lifting of a flat torsion free connection (that could be carried over to the algebraic setting) and the geodesic exponential mapping that in turn involves solving ordinary differential equations.

For general actions and $k=\mathbb{C}$ there are further obstructions for lifting of automorphisms of $X / G$ that are related to the fundamental group of $X / G$.

\section{Appendix}

Here we give some details of the proof of Theorem 2.2.1 for finite $G$.

This proof is based on the following preliminary results all proved in $[\mathrm{Lu}]$. 
(a) Let $X$ be a normal affine variety with a faithful action of $G$. Let $H$ be a subgroup of $G$ and let $x$ be a point of $X$. Then the natural map $\alpha: X / H \rightarrow$ $X / G$ is étale at $\pi_{X, H}(x)$ if and only if $H \supseteq G_{x}$.

(b) Let in addition $\varphi: X \rightarrow Y$ be a finite equivariant morphism to another normal affine $G$-variety. If $\varphi$ is étale at $x$, and if $G_{\varphi(x)}=G_{x}$, then the map $\varphi / G: X / G \rightarrow Y / G$ is étale at $\pi_{X, G}(x)$.

(c) If $x \in X_{\mathrm{sm}}$, then there exists a $G_{x}$-equivariant morphism $\varphi: X \rightarrow T_{x, X}$ such that $\varphi$ is étale at $x$ and $\varphi(x)=0$.

Statement (a) is [Lu, Lemme 2], proved by references to results of commutative algebra; (b) is [Lu, Lemme 1], proved on p. 92, Case (B); and (c) is [Lu, Lemme, p. 96] (one can assume that $X$ is a $G$-stable subvariety of a $G$-module $k^{n}$ and $x=0$, cf. [PV, Theorem 1.5]; if $\pi: k^{n} \rightarrow T_{x, X}$ is the projection parallel to a $G_{x}$-stable complement to $T_{x, X}$, then clearly one can take $\varphi=\left.\pi\right|_{X}$.)

Note that (a) implies that $X \rightarrow X / G$ is étale whenever $G$ acts freely.

To prove Theorem 2.2.1 (i), note that $X / G_{x} \rightarrow X / G$ is étale at $\pi_{X, G_{x}}(x)$, by (a). So there exists a $G_{x}$-invariant open subset $S \subseteq X$ such that $S / G_{x} \rightarrow X / G$ is étale. Then its base change $X \times_{X / G} S / G_{x} \rightarrow X$ is étale as well. But the composition of $\psi: G *_{G_{x}} S \rightarrow X \times_{X / G} S / G_{x}, G_{x} \cdot(g, s) \mapsto\left(g \cdot s, G_{x} \cdot s\right)$, with this base change is just the natural map $G *_{G_{x}} S \rightarrow X$, which is obviously étale (since it factors as the open immersion $G *_{G_{x}} S \rightarrow G *_{G_{x}} X$, followed by the projection $\left.G *_{G_{x}} X=G / G_{x} \times X \rightarrow X\right)$. Thus, $\psi$ is étale as well. On the other hand, the (set-theoretic) fiber of $\psi$ at $\tilde{x}=G_{x} \cdot(e, x) \in G *_{G_{x}} S$ is the unique point $\tilde{x}$. Thus, shrinking $S$, we may achieve that $\psi$ is an isomorphism.

To prove Theorem 2.2.1 (ii), consider $\varphi$ from (c). Then $\varphi / G_{x}: X / G_{x} \rightarrow$ $T_{x, X} / G_{x}$ is étale at $\pi_{X, G_{x}}(x)$, by (b). By the preceding argument, the natural map $X \rightarrow X / G_{x} \times_{T_{x, X} / G_{x}} T_{x, X}$ is étale at $x$, and its fiber there is the unique point $x$. This yields a $G_{x}$-invariant open subset $U \subseteq X$ such that $U / G_{x} \rightarrow$ $T_{x, X} / G_{x}$ is étale, and $U \rightarrow U / G_{x} \times_{T_{x, X} / G_{x}} T_{x, X}$ is an isomorphism.

\section{REFERENCES}

[BR] P. Bardsley, R. W. Richardson, Étale slices for algebraic transformation groups in characteristic p, Proc. Lond. Math. Soc., I, Ser. 51 (1985), 295-317.

[B] M. Brion, Differential forms on quotients by reductive group actions, Proc. AMS 126 (1998), 2535-2539.

[GR] R. C. Gunning, H. Rossi, Analytic Funstions of Several Complex Variables, Prentice-Hall, Inc., Englewood Cliffs, N. J., 1965.

[He] M. Hervé, Several Complex Variables, Oxford University Press, Bombay, 1963.

[Hu] M. Hunziker, Classical invariant theory for finite reflection groups, Transformation Groups 2 (1997), no. 2, 147-163.

[KLM] A. Kriegl, M. Losik, P. W. Michor, Tensor fields and connections on holomorphic orbit spaces of finite groups, in preparation (2001).

[Lo] M. Losik, Lifts of diffeomorphisms of orbit spaces for representations of compact Lie groups, Geom. Dedicata 88 (2001), 21-36.

[Lu] D. Luna, Slices étales, Bull. Soc. Math. France, Memoire 33 (1973), 81-105.

[M] P. W. Michor, Basic differential forms for actions of Lie groups, Proc. AMS 124 (1996), 1633-1642; II, Proc. AMS 125 (1997), 2175-2177. 
INVARIANT TENSOR FIELDS FOR ALGEBRAIC FINITE GROUP ACTIONS 27

[PV] V. L. Popov, E. B. Vinberg, Invariant Theory, Algebraic Geometry, IV, Encycl. of Math. Sci., vol. 55, Springer-Verlag, Heidelberg, 1994, pp. 123-284.

[Sch] G. W. Schwarz, Lifting smooth homotopies of orbit spaces, Publ. Math. IHES 51 (37-136), 1980.

[Sh] I. R. Shafarevich, Basic Algebraic Geometry, Springer-Verlag, Berlin, Heidelberg, 1994.

[Si] J. H. Silverman, The Arthmetic of Elliptic Curves, Springer-Verlag, New York, Berlin, 1986.

[So] L. Solomon, Invariants of finite reflection groups, Nagoya Math. J. 22 (1963), $57-64$.

M. Losik: Saratov State University, ul. Astrakhanskaya, 83, 410026 Saratov, RUSSIA

E-mail address: LosikMV@info.sgu.ru

P. W. Michor: Institut für Mathematik, Universität Wien, Strudlhofgasse 4, A-1090 Wien, Austria; and: Erwin Schrödinger Institut für Mathematische Physik, Boltzmanngasse 9, A-1090 Wien, Austria

E-mail address: Peter.Michor@esi.ac.at

V.L. Popov: Steklov Mathematical Institute, Russian Academy of Sciences, Gubkina 8, Moscow, 117966, Russia

E-mail address: popov@ppc.msk.ru 